\title{
The Many Faces of the Heterotic Vortex
}

\author{
S. Bolognesi \\ FTPI \& U of M
}

CROSSING THE BOUNDARIES --- May 14, 2009 
Plan of the Talk

* Intro on non-Abelian Vortex

$\% \quad w=2$ \& $w=1$ in $3+1$

* Results of arxiv/0903.3422

* Work in furogress with M,S.

\& M.S.\& M.V. arxiv $/ 0905.1664$ 
What is the non-Abelian vortex

BASIL INGREDIENTS

$\otimes U(n)=\frac{S U(n) \times U(1)}{\mathbb{Z}_{n}}$

$\forall n_{f} \geqslant n_{c}$ quarks

$\checkmark$ CF phase:

$$
\langle q\rangle=\left(\begin{array}{lll}
\sqrt{3} & & 0 \\
& & \\
& \sqrt{2} &
\end{array}\right)
$$


What is the non-Abelian vortex

BasIL IngREDIENTS

$\otimes U(n)=\frac{S U(n) \times U(1)}{\mathbb{Z}_{n}}$

$C P\left(n_{-}-1\right)$

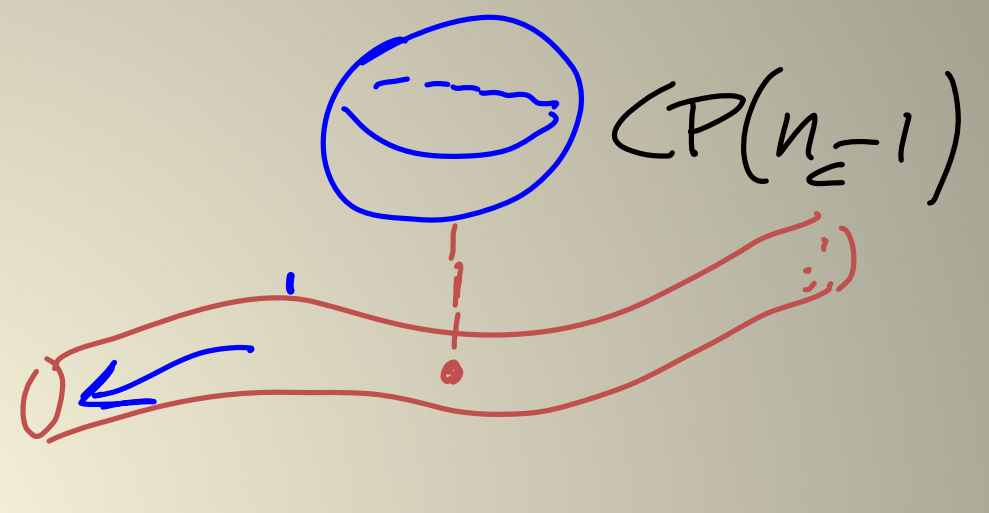

$* n_{f} \geqslant n_{c}$ quarks

Seneralization

of orchrnary ANO

\& F thase:

$\langle q\rangle=\left(\begin{array}{lll}\sqrt{3} & & 0 \\ \hdashline & & \\ & \sqrt{3} & \end{array}\right)$

- So(n) magflux

- Modul Spece 
Why bother about it ...

\& Is there...

* Ponfines non-Ablion monopoles

* Squeexing $3+1 \longrightarrow 1+1$

* QCD dual suferconoluctor 
The theory: $\mathrm{N}=2$ Super-QCD

$$
\begin{aligned}
& \begin{array}{l}
U(n) \text { gauge } \\
\tau=\frac{4 \pi i}{g^{2}}+\frac{\theta}{2 \pi}
\end{array} \quad \lambda^{A \mu} \psi \quad \phi^{+\mu_{f}} \quad q_{\frac{\psi_{\pi}^{+}}{q^{d}}}
\end{aligned}
$$

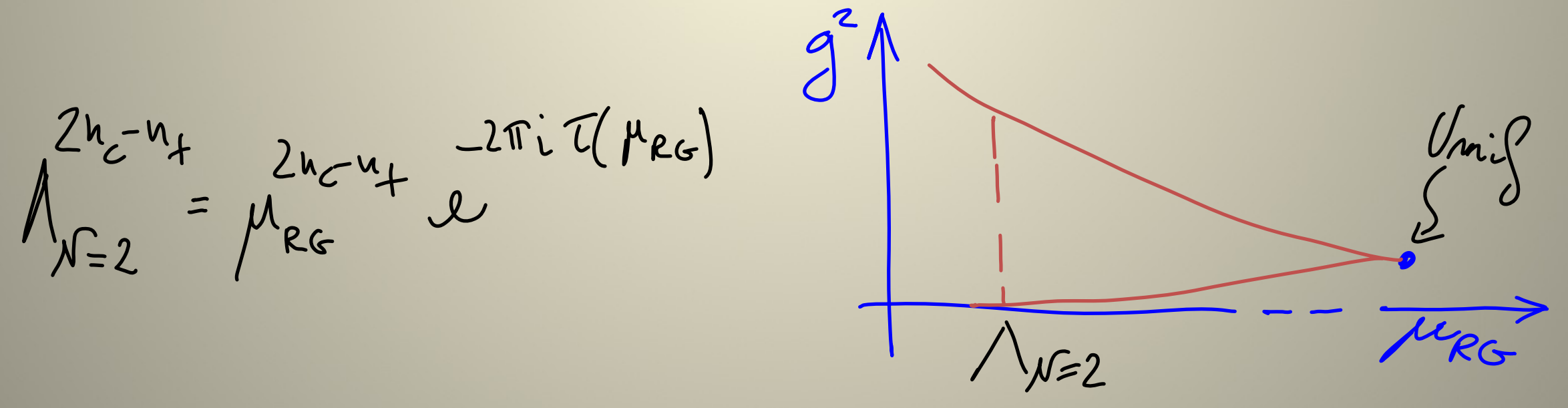


The theory: $\mathrm{N}=2$ Super-QCD

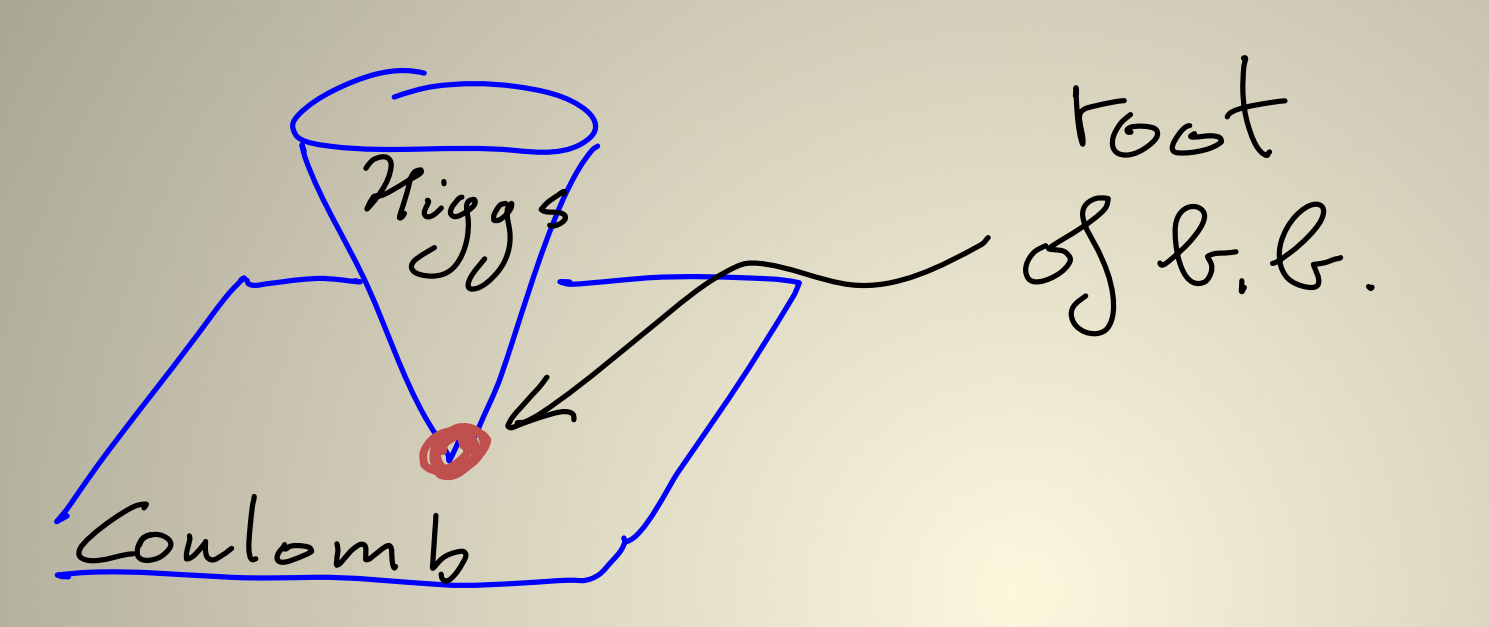

Moduli space

$$
\Lambda_{N=2}^{2 n_{c}-n_{t}}=\mu_{R G}^{2 n_{C}-u_{t}} e^{-2 \pi i \tau\left(\mu_{R G}\right)}
$$

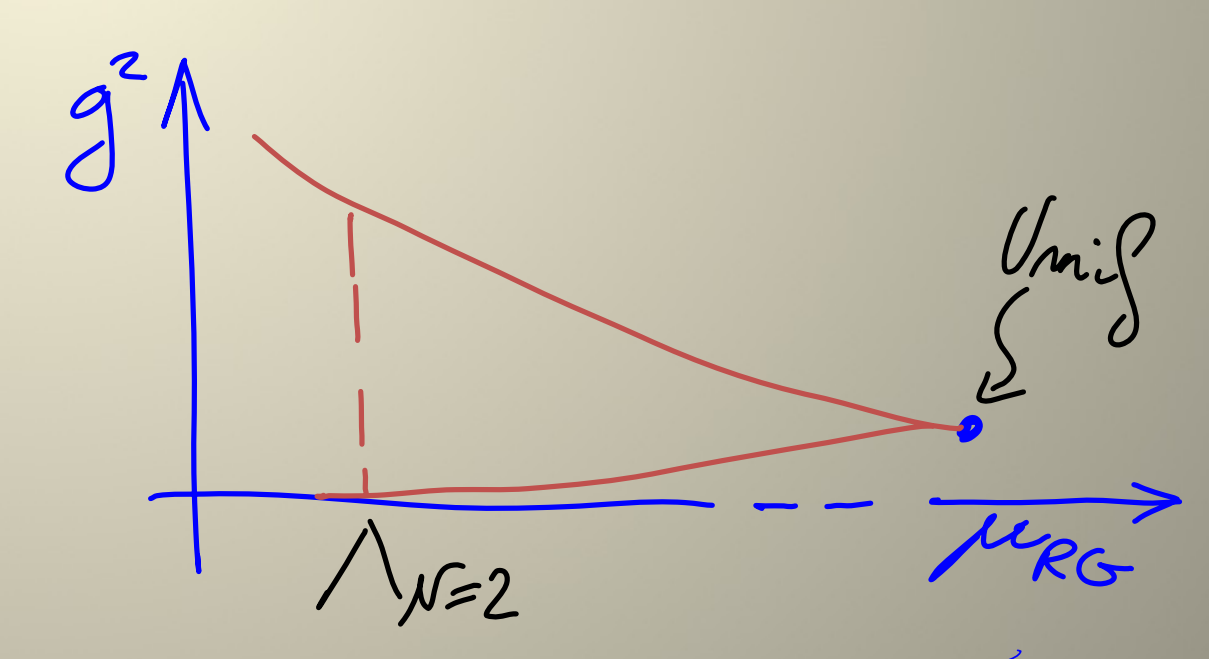


Add FI term, what happens ... for $U(1) \quad-2 \xi \int d^{2} \theta d^{2} \dot{\theta} t_{2} V$ $w=2$ UNBROKEN! but SU(2)R $\rightarrow U(1)_{J}$ 
Add FI term, what happens ... for $U(1) \quad-2 \xi \int d^{2} \theta d^{2} \dot{\theta} t_{2} V$ $w=2$ UNBROKEN! but SU(2)R $\rightarrow U_{(1)}$

$$
\langle q\rangle=\left(\begin{array}{ccc}
\sqrt{2 \xi} & & 0 \\
\vdots & & \\
& \sqrt{2 \xi} &
\end{array}\right) \quad\langle\tilde{q}\rangle=0
$$

Color-Flovo locking phase $\operatorname{su}\left(n_{c}\right) \times \operatorname{su}\left(n_{f}\right) \rightarrow \operatorname{su}\left(n_{c}\right)_{c+f}+\ldots$ 
Non-Abelian vortex here ...

WEAK COUPLING $\sqrt{3} \gg \Lambda_{N=2} \quad n_{c}=n_{f}=n$

$$
\alpha=-\frac{1}{2 g^{2}} t_{2}(F F)+(D q)^{+} D_{q}-\frac{g^{2}}{4}+\frac{1}{2}\left(9 q^{+}-2 \xi \frac{11}{)^{2}}\right)^{2}
$$


Non-Abelian vortex here ...

WEAK COUPLING $\sqrt{\xi} \gg \Lambda_{N=2} \quad n_{c}=n_{f}=n$

$$
\alpha=-\frac{1}{2 g^{2}} t_{2}(F F)+(D q)^{+} D_{q}-\frac{g^{2}}{2}+\frac{1}{2}\left(99^{+}-23 \frac{11}{)^{2}}\right)^{2}
$$

Topology $\pi_{1}(U(1))=\mathbb{Z}=n$ times $\pi_{1}\left(\frac{s u(n) \times U(1)}{\mathbb{Z}_{n}}\right)$

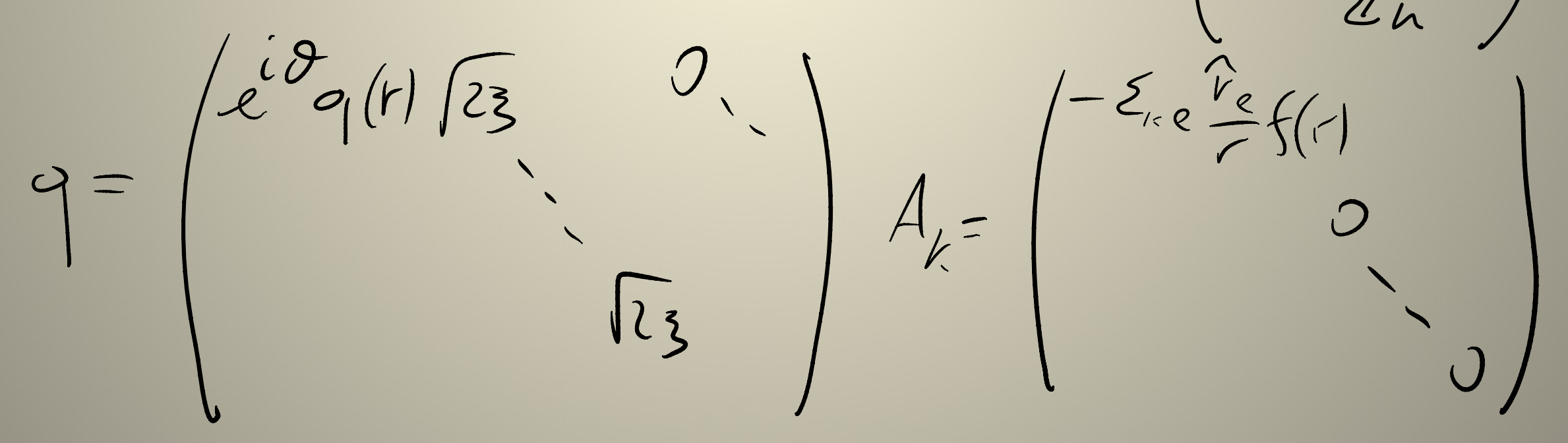


Moduli space \& Effective action

$$
\begin{aligned}
& \operatorname{su}(n)_{c+f} \longrightarrow S U(n-1) \times U(1) \\
& \text { * Internal Model } \\
& \text { * } \vec{B} \operatorname{flu} x \operatorname{su}(n) \\
& \text { Coset }=C P(n-1) \\
& \begin{array}{c}
0 \\
0 \quad ! \\
0 \quad 1
\end{array} \\
& \beta=\frac{2 \pi}{g^{2}}=\frac{n}{4 \pi} \log \frac{\sqrt{3}}{\Lambda_{3+1}} \\
& \Lambda_{C p}=\Lambda_{3+1}
\end{aligned}
$$




$$
\begin{aligned}
& \text { SUSY } N=(2,2) \text { in } 1+1 \\
& 11 \quad \begin{array}{l}
Q^{\prime}=\left(Q_{L}^{1}, Q_{R}^{\prime}\right) \\
Q^{2}=\left(Q_{L}^{2}, Q_{R}^{2}\right)
\end{array} \\
& S=(2,2)=\left(Q_{L}^{2}, Q_{R}^{1}\right) \\
& \text { BY THE VORTE? } \\
& \zeta_{L}^{z} \zeta_{R} \quad \xi_{L}^{l} \varphi^{l} \xi_{R}^{l}
\end{aligned}
$$


An experiment ...

$$
\xi=0
$$

Coulomb Phase $\quad M_{\text {BPS }}=|Z|$<smiles>CCOCCCCCCCOC</smiles> 
An experiment ...

$$
\begin{aligned}
& 3 \gg \wedge \\
& \text { Higgs thase } \quad M_{B P S}^{3+1}=M_{\text {BPS }}^{1+1} \\
& \text { SQUEEZ }
\end{aligned}
$$

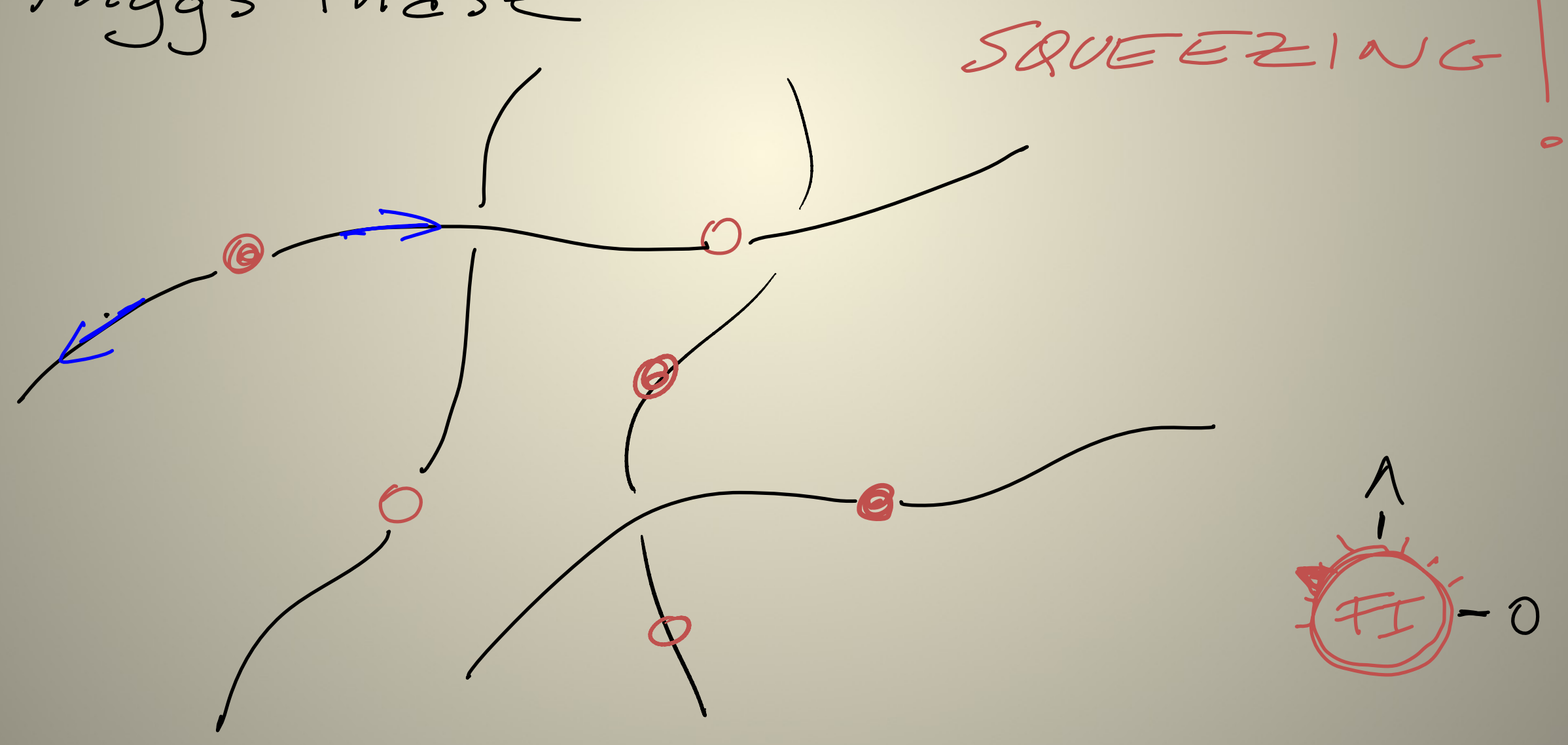


$\mathrm{N}=1$ mass deformation

$$
\begin{aligned}
& \int d^{2} \theta \underbrace{\sqrt{2} \mu t_{2} \Phi^{2}}_{W(\Phi)}+h \cdot c \\
& \Lambda_{N=1}^{3 n_{c}-n_{f}}=\mu^{k} \Lambda_{N=2}^{2 u_{c}-n_{f}}
\end{aligned}
$$


$\mathrm{N}=1$ mass deformation

$$
\int d^{2} \theta \underbrace{\sqrt{2} \mu t_{2} \Phi^{2}}_{w(\Phi)}+h \cdot c
$$

Coincidence $W^{\prime}(m)=0$

SECTRUM \& Weak coupling

$$
m_{A_{\mu}}=g \sqrt{2 \xi} \quad \underbrace{m_{h}=g \mu \quad m_{l}=\xi / \mu}_{\text {la sse } \mu}
$$


The Coincidence ...

Withouf FI

$$
\begin{gathered}
W=t_{2} W(\phi)+\widetilde{Q}(\Phi-m) Q \\
W^{\prime}(a)=0
\end{gathered}
$$

- Hugs $\tilde{q} q=\omega^{\prime}(m) \quad \phi=m$

- Couloub $q=\hat{q}=0 \quad \phi=a$ Coulomb

$$
M \rightarrow O \quad+Q, \widetilde{Q} \text { mostess }
$$


The Coincidence ...

Without FI

WITH II

$$
\begin{gathered}
D=t_{2} w(\phi)+\widetilde{Q}(\Phi-m) Q \\
W^{\prime}(a)=0
\end{gathered}
$$

- Hugs $\quad \tilde{q} q=\omega^{\prime}(m) \quad \phi=m$

- Coulomb $q=\hat{q}=0 \quad \phi=a$ Coulomb

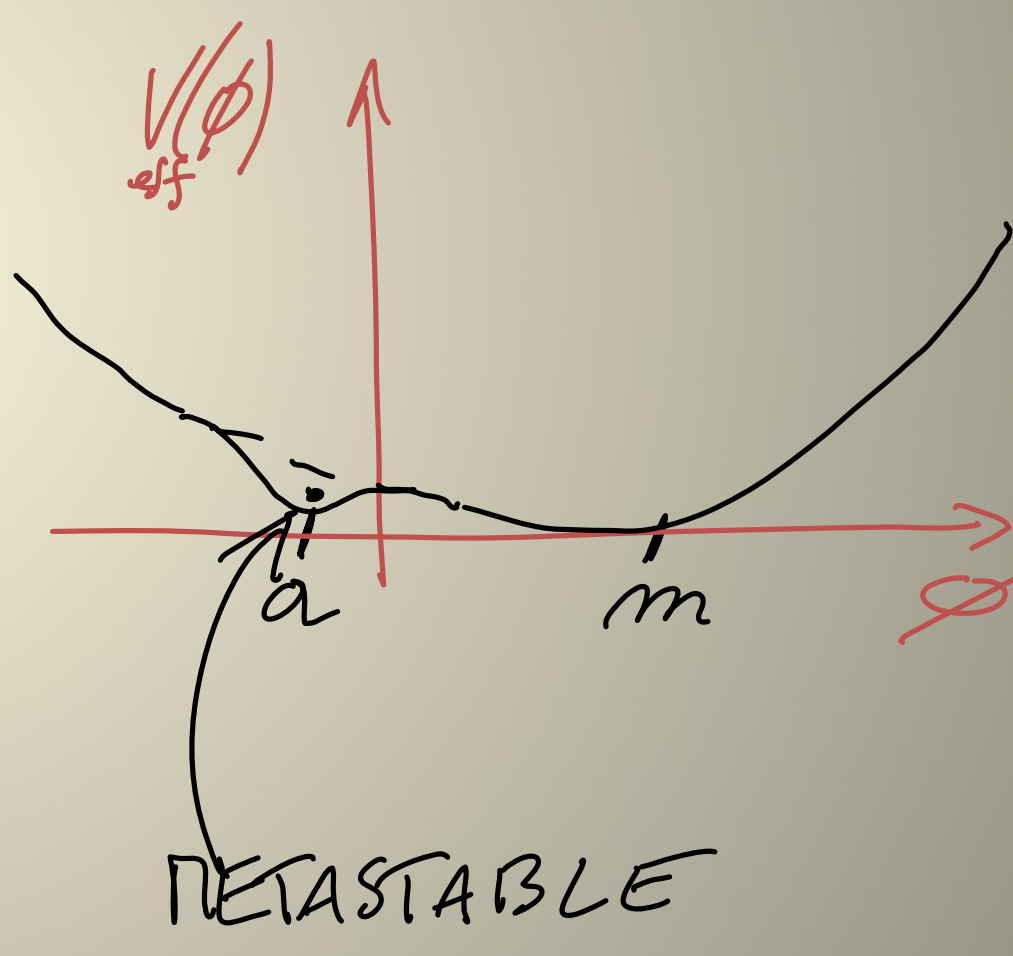

$M \rightarrow a$ $+Q, \widetilde{Q}$ mosses 
Moduli space enhancement

$$
\begin{array}{r}
\quad m_{l}=3 / \mu \rightarrow 0 \\
W_{\text {off }}=\frac{1}{\mu}+n \text { Q } \hat{Q} Q \tilde{Q}
\end{array}
$$

"Pure" N=1 SQCD has No Fo term

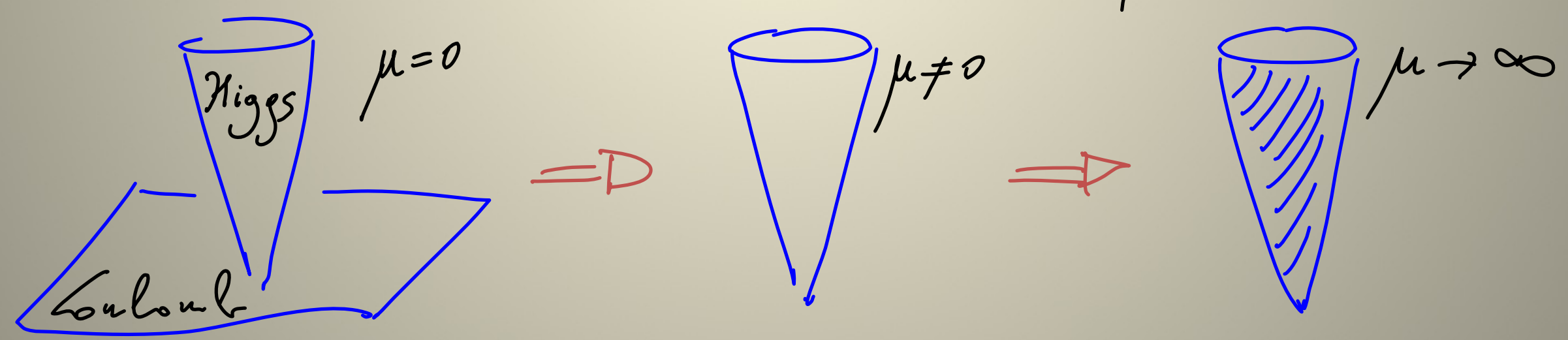


Heterotic $\mathrm{N}=(0,2)$ deformation

* String reman BPS if W/(Mu)=0

* FirT Myp. enhancenert of sosy vo $v=(0,2)$ of $C P(n-1)$

* H.T. oby $W_{1+1} \propto \frac{1}{\sqrt{3}} W_{3+1}$

$\int d^{2} x d^{2} g_{R} \cap W_{m ! l}^{\prime}\left(\varepsilon_{1}\right)+h c$ 
Fermionic zero modes (1)

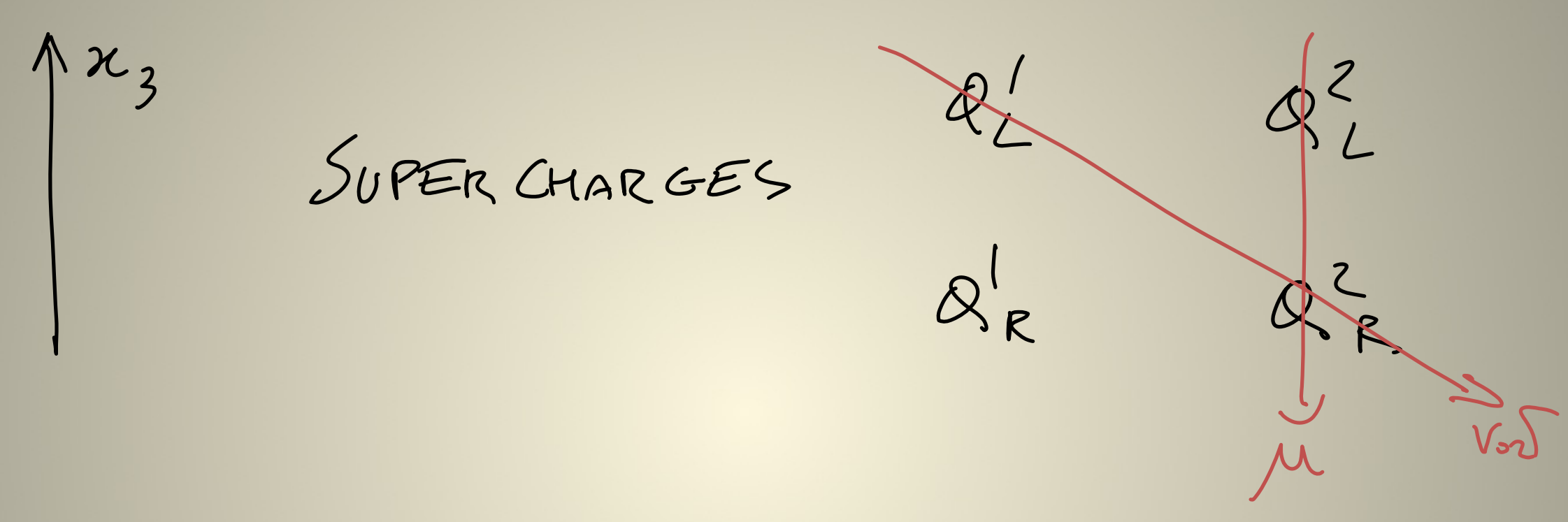


Fermionic zero modes (1)

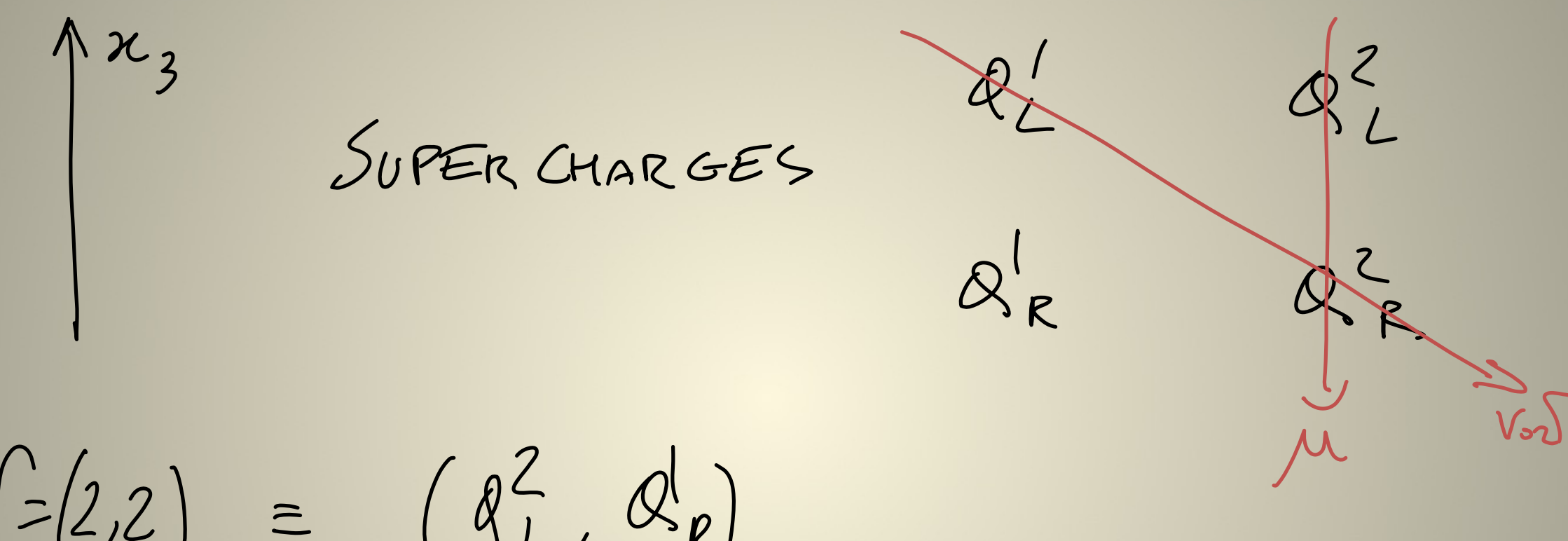

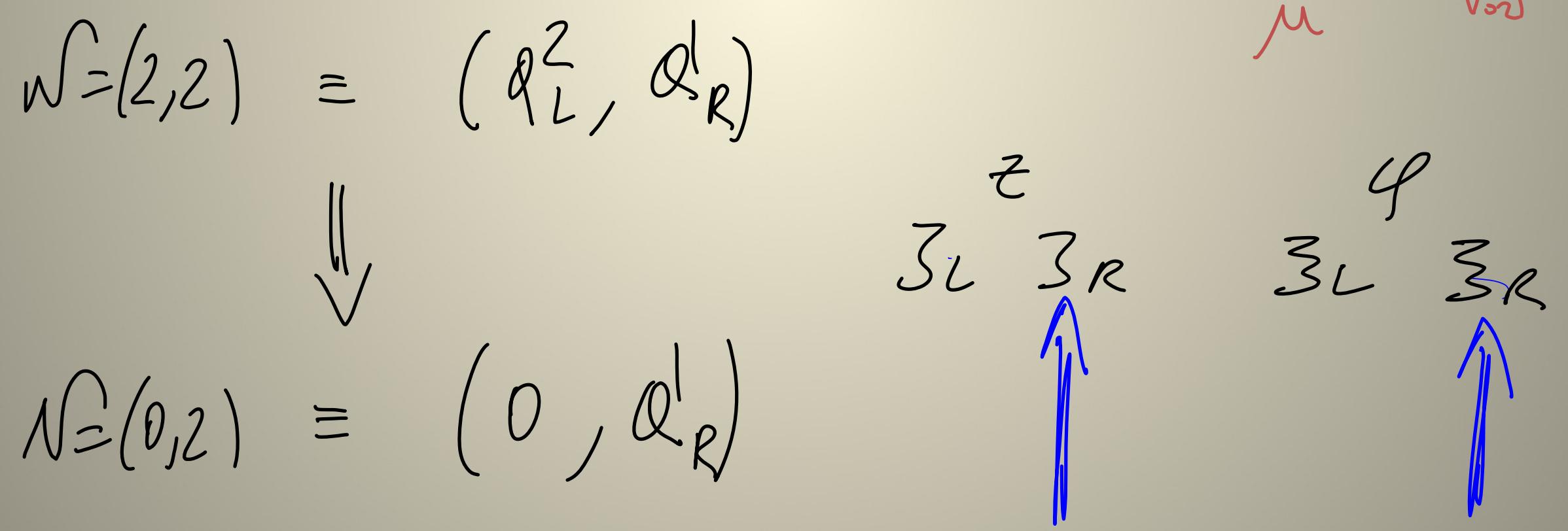


Fermionic zero modes (2)

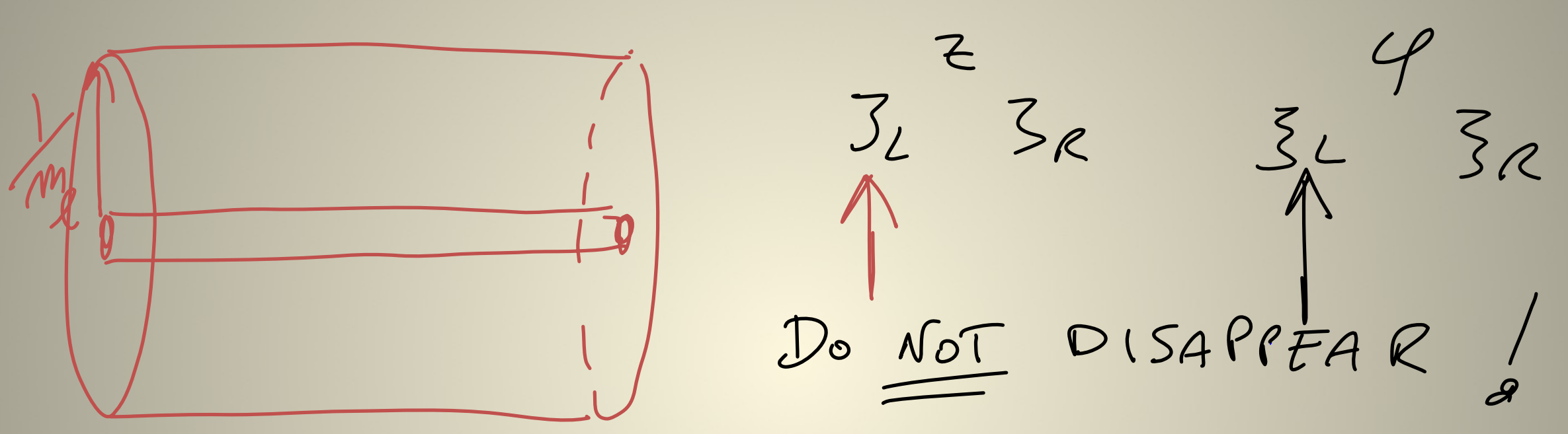

$$
\Psi_{L} \propto \frac{e^{-m_{e} r}}{\sqrt{r}} \quad \text { THEY BECOME }
$$

Non Normalizable! 
Large $\mathrm{n}$ solution \& DSB

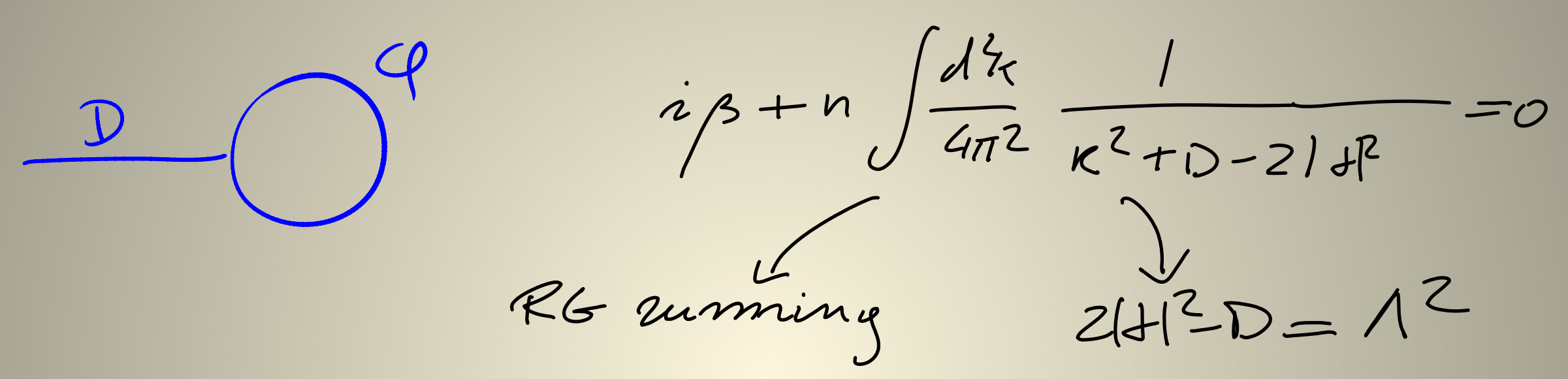


Large $\mathrm{n}$ solution \& DSB

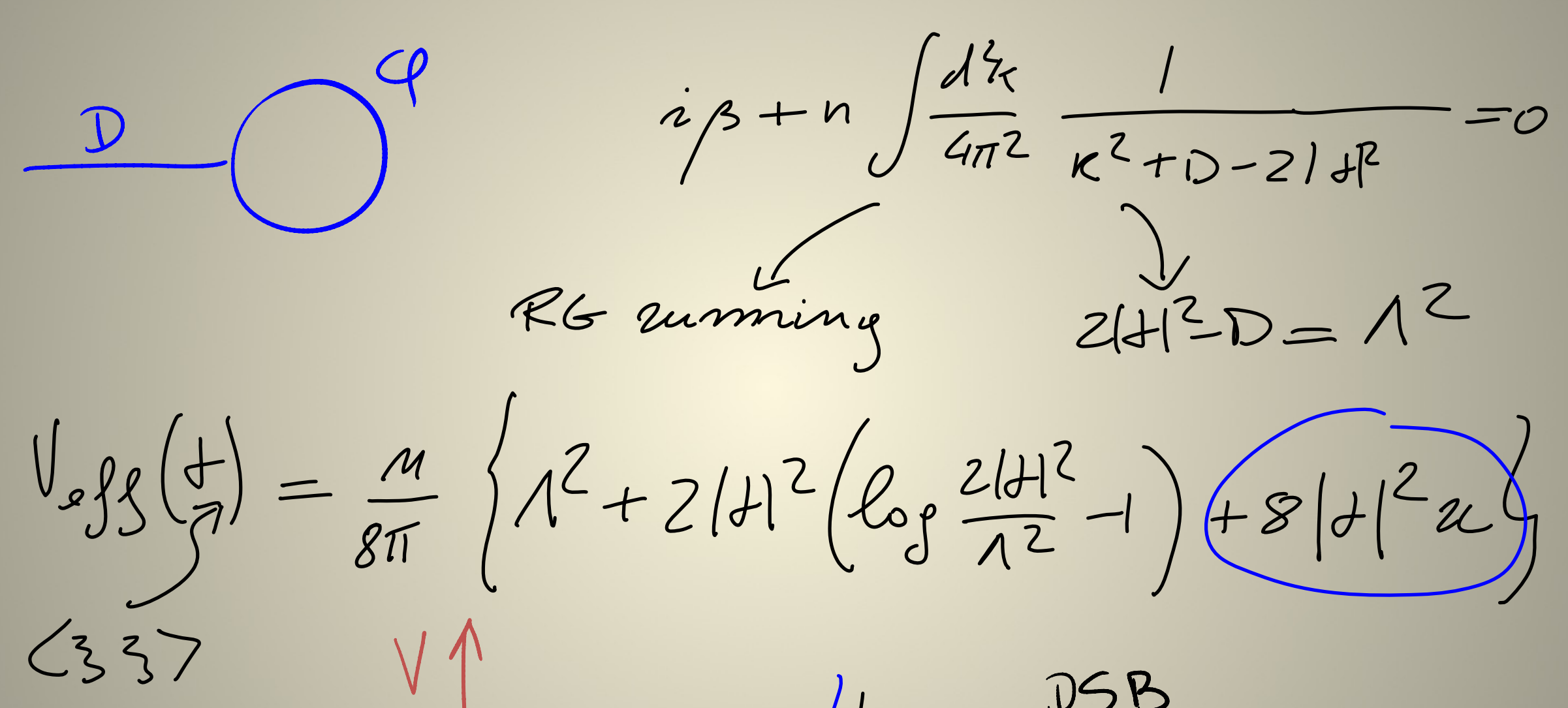

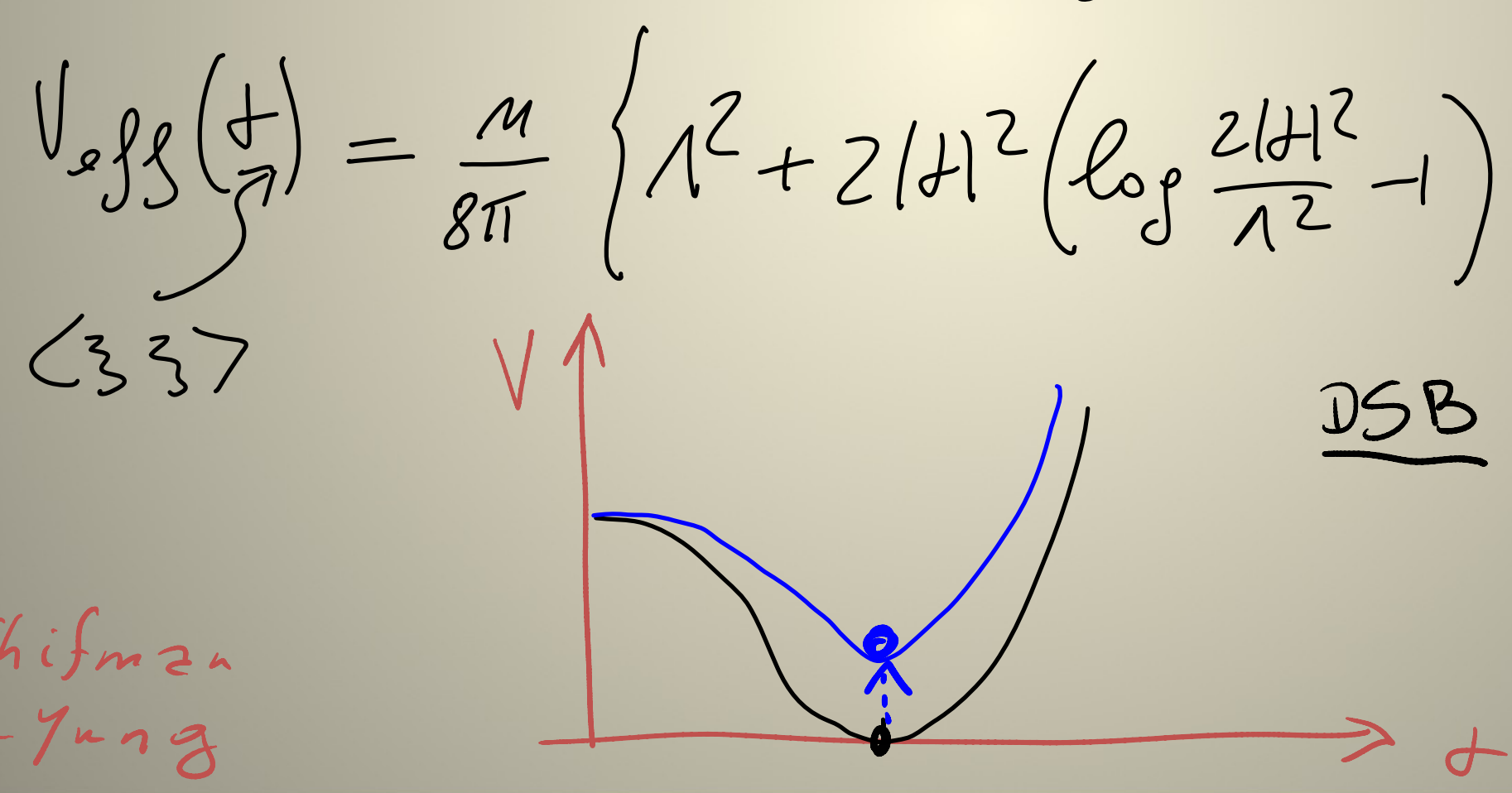


New stuff ...

* Explor Yaramete Space $(\mu, \xi) \quad\left\{\begin{array}{l}\text { DSB... } \\ \text { degenerecy... }\end{array}\right.$

$\alpha \mu \operatorname{rr}\left(\frac{\Phi}{2}-a \Phi\right)$ LINEAR TERT

Conjectured NEW CFT As $\quad \sqrt{\xi} \gg 1, \mu \rightarrow \infty$ 


\section{Exploring the parameter space}

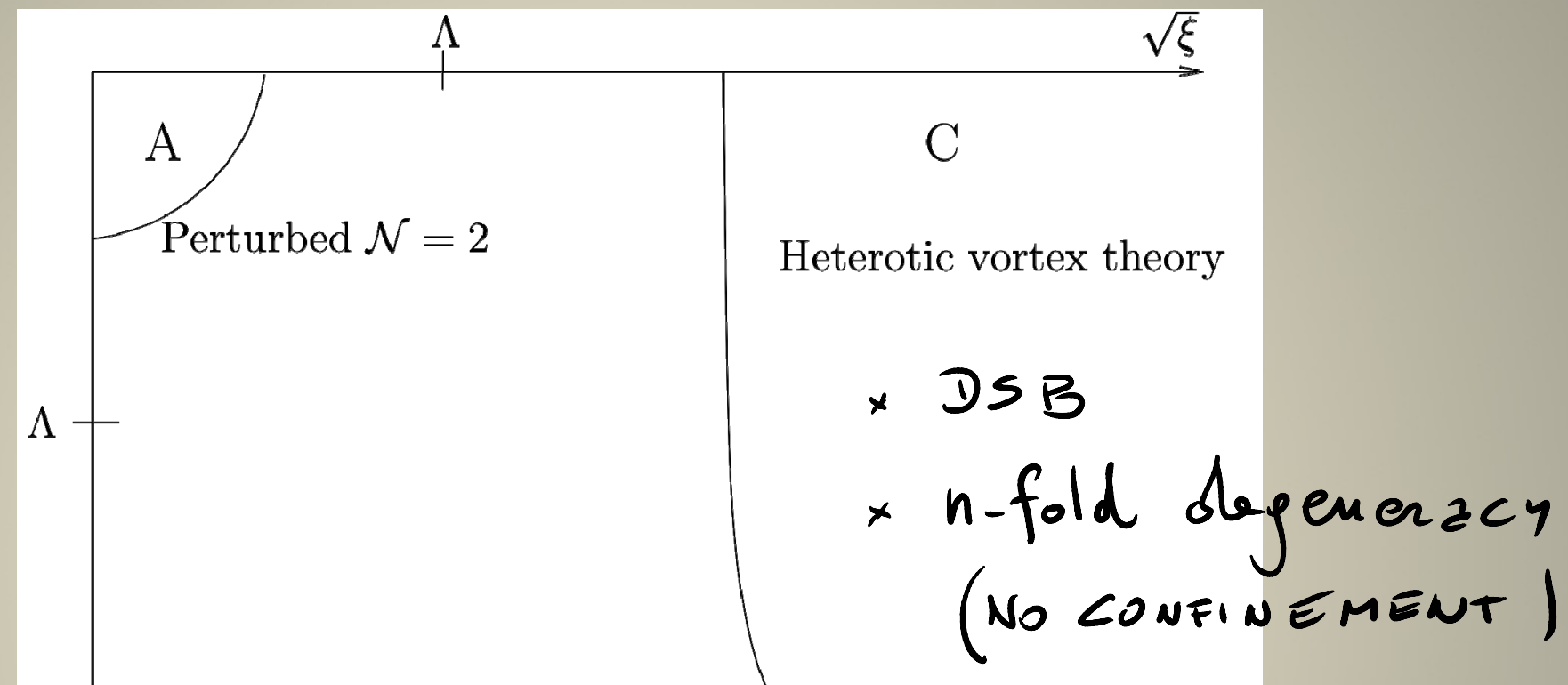

STRONG

WEAK $(3+1)$ 
(A): SW solution

$$
\begin{aligned}
& y^{2}=\frac{1}{4} \operatorname{det}(z-\phi)^{2}-\Lambda^{m} z^{n} \\
& =\frac{1}{4}\left(z^{n}-\Lambda^{n}\right)^{2}
\end{aligned}
$$

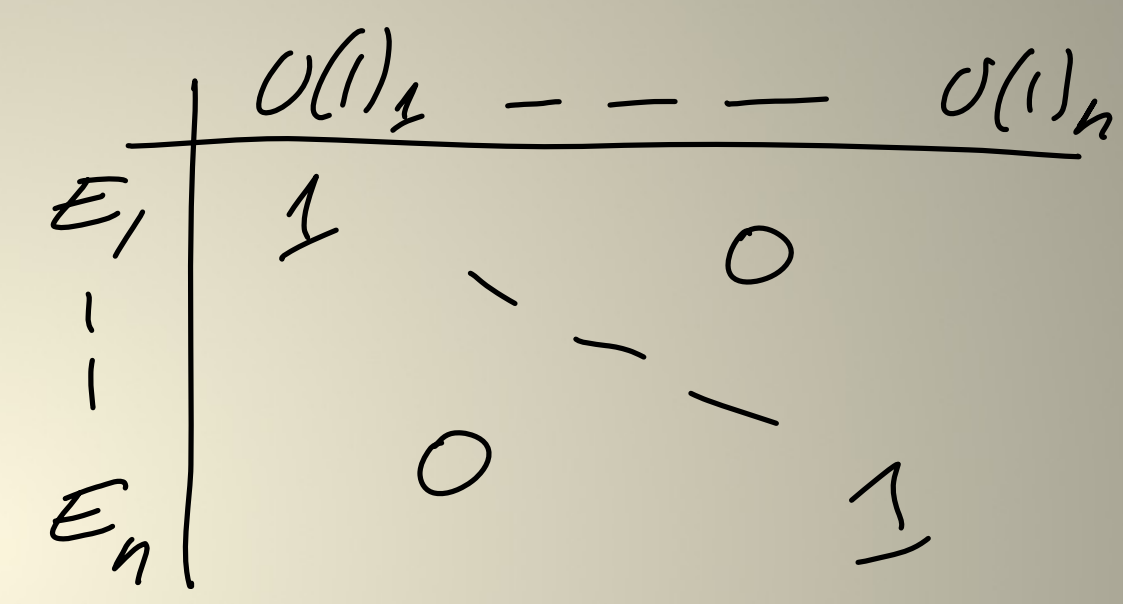

Roots

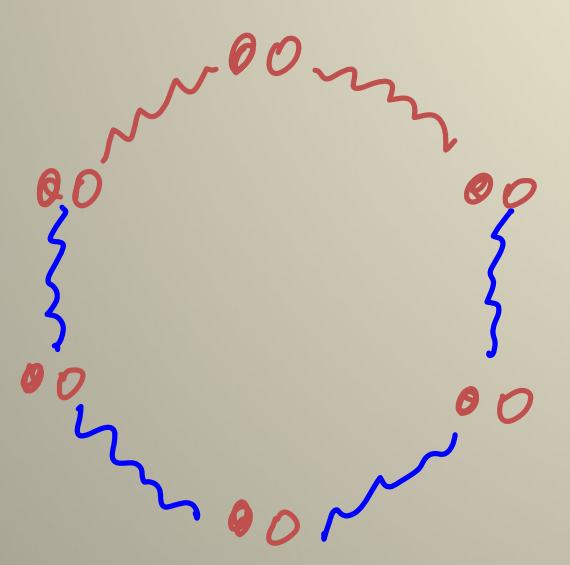


(A): SW solution

$$
\begin{aligned}
& y^{2}=\frac{1}{4} \operatorname{det}(z-\phi)^{2}-\Lambda^{m} z^{n} \\
& =\frac{1}{4}\left(z^{n}-\Lambda^{n}\right)^{2}
\end{aligned}
$$

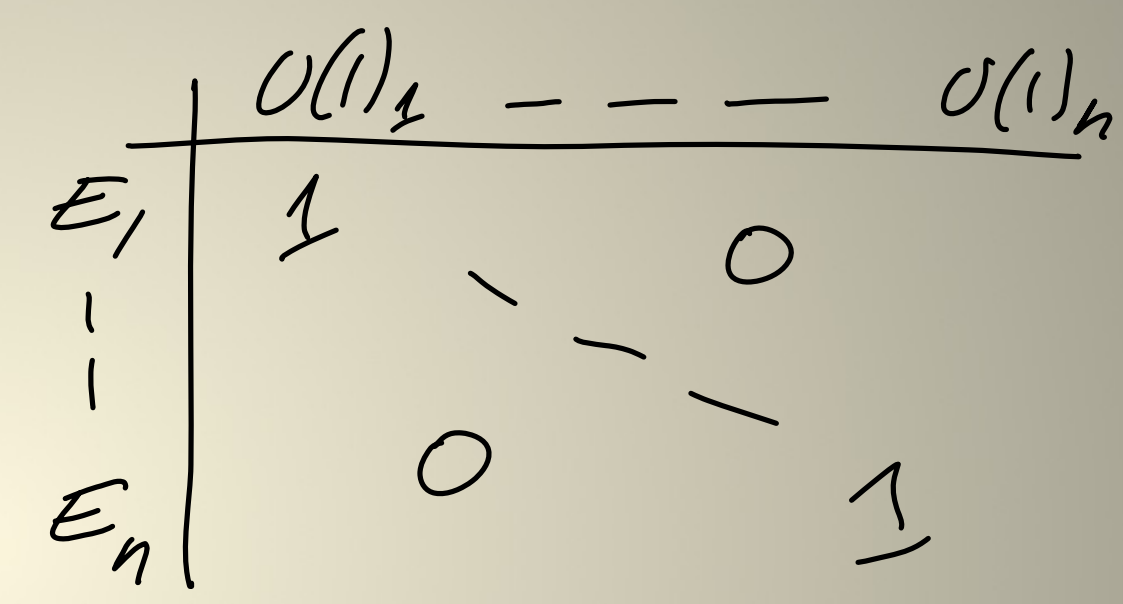

Roots

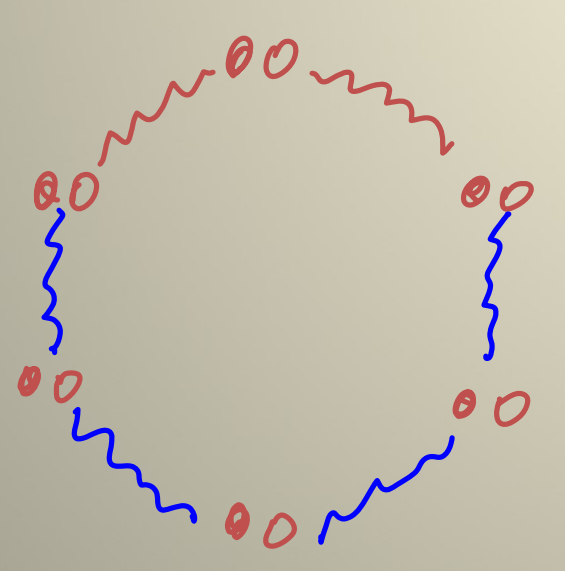

Maca Cunve

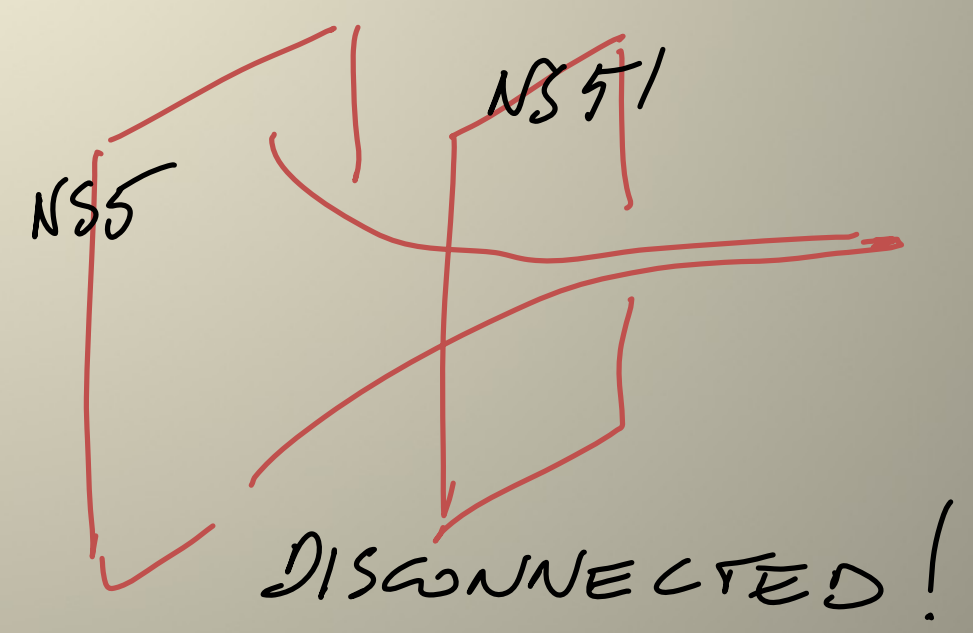


(A): Vortices here

$$
\begin{aligned}
& T_{j \propto} \sqrt{\left|w^{\prime}(200 t,)\right|^{2}+3^{2}} \\
& \simeq \xi+\sim \frac{\mu^{2} \Lambda^{2}}{\xi}+\ldots
\end{aligned}
$$

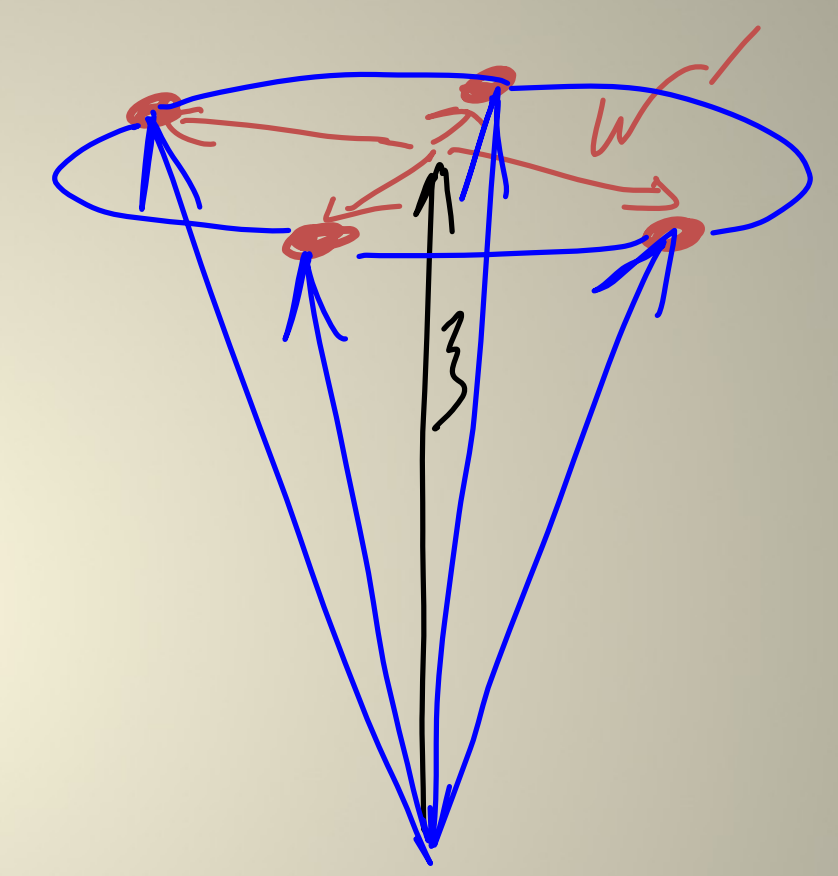


(A): Vortices here

$$
\begin{aligned}
& T_{j} \sqrt{\left|w^{\prime}\left(200 t_{0}\right)\right|^{2}+\xi^{2}} \\
& \simeq \xi+\sim \frac{\mu^{2} \Lambda^{2}}{\xi}+\ldots
\end{aligned}
$$

Explain:

- DSB \& degenerecy $(a=0)$

No leganerecy $a \neq 0$

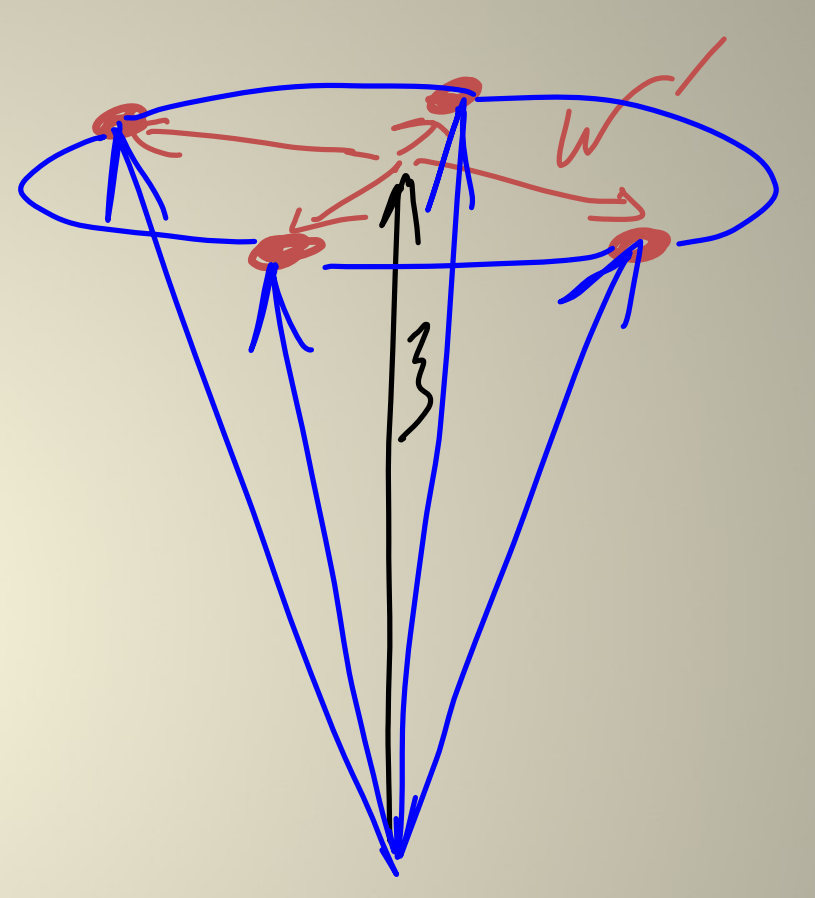

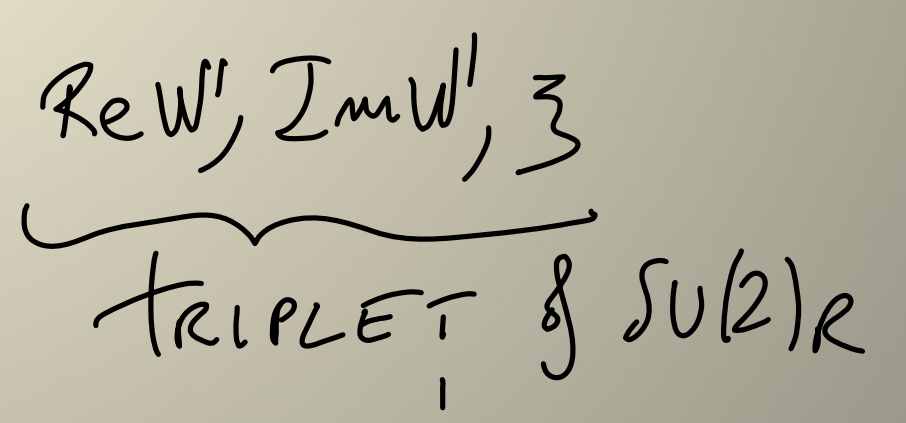

- SUSY RESTORATION $a=1 e^{\frac{i 2 \pi K K}{n} !}$ 
(B) Perturbed $\mathrm{N}=1$

FIRST $N=1$ SQCD

$$
\begin{aligned}
& M=\widetilde{Q} Q \\
& \operatorname{det} M-B \widetilde{B}=0 \\
& B=\epsilon Q--Q
\end{aligned}
$$

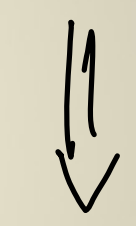

$$
\begin{aligned}
& \widetilde{B}=\epsilon \widetilde{Q}_{---Q} \\
& \operatorname{det} \pi-B \tilde{B}=\Lambda^{2 m} \\
& \text { m "BASES" } \\
& \text { Where } \widetilde{B}=0 \\
& M=\frac{1}{m \times m}^{2} \Lambda^{\frac{2 \pi i k}{h}}
\end{aligned}
$$


(B): Effective superpotential

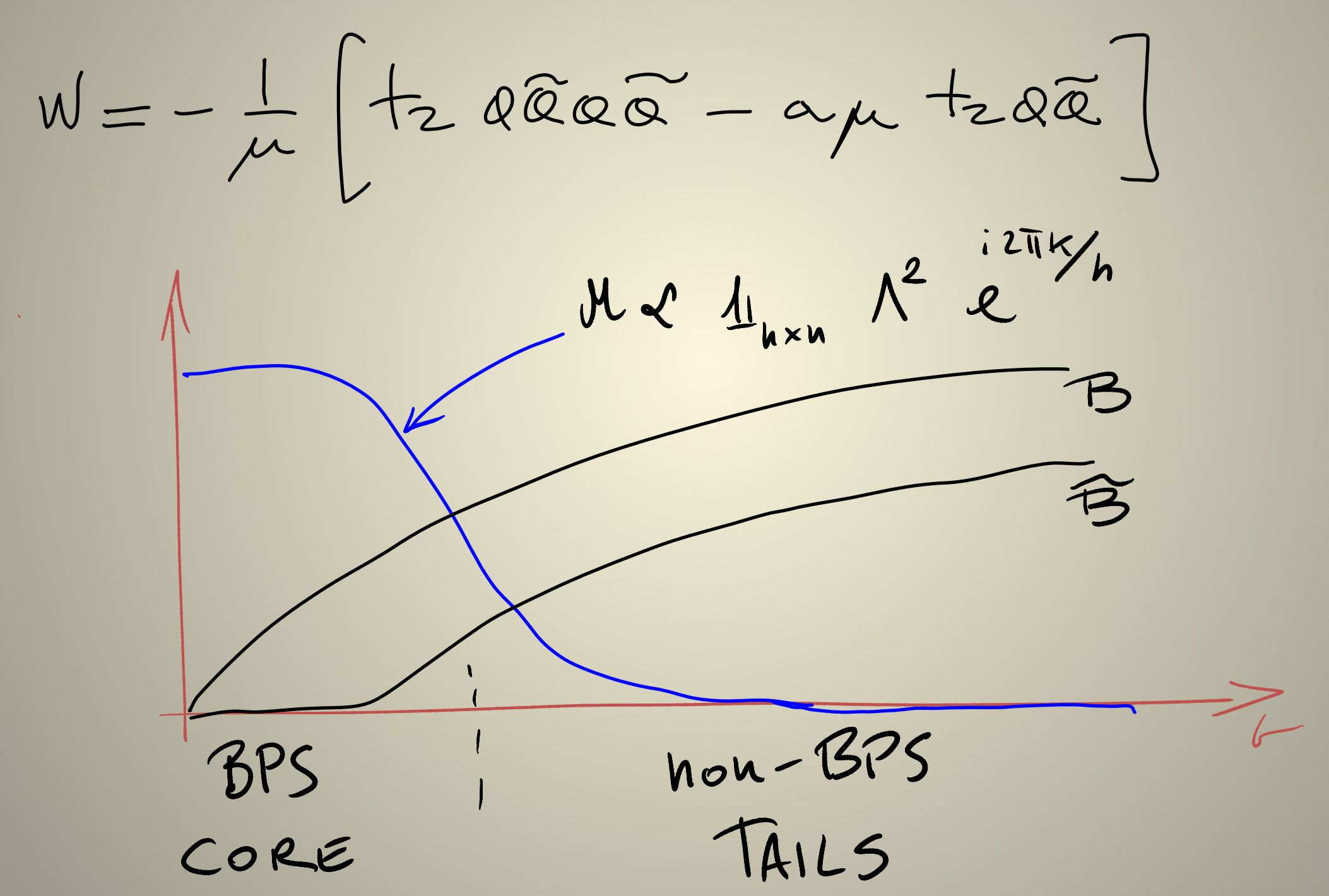


(C): Heterotic vortex theory

$$
\begin{gathered}
V_{\text {eff }}(t)=\frac{\mu}{8 \pi}\left\{\Lambda^{2}+\partial^{2}\left(\log \frac{\partial^{2}}{\lambda^{2}}-1\right)+8|\alpha-a|^{2} u\right\} \\
a=\frac{\Lambda}{\sqrt{2}} \quad \text { SUSY IS NOT } \\
\text { BROKEN }
\end{gathered}
$$

- Susy is actuacly Enhancen $w=(2,2) \quad$ SPECTRUM 
(D): Quantum Heterotic Vortex

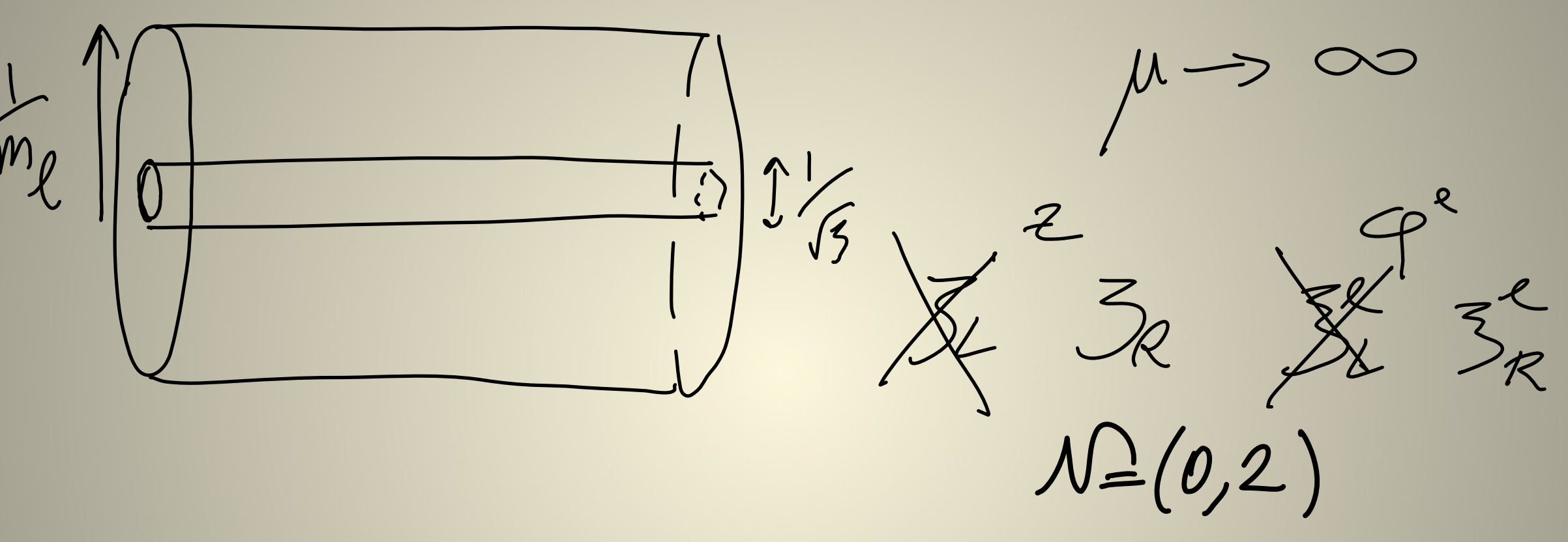


(D): Quantum Heterotic Vortex

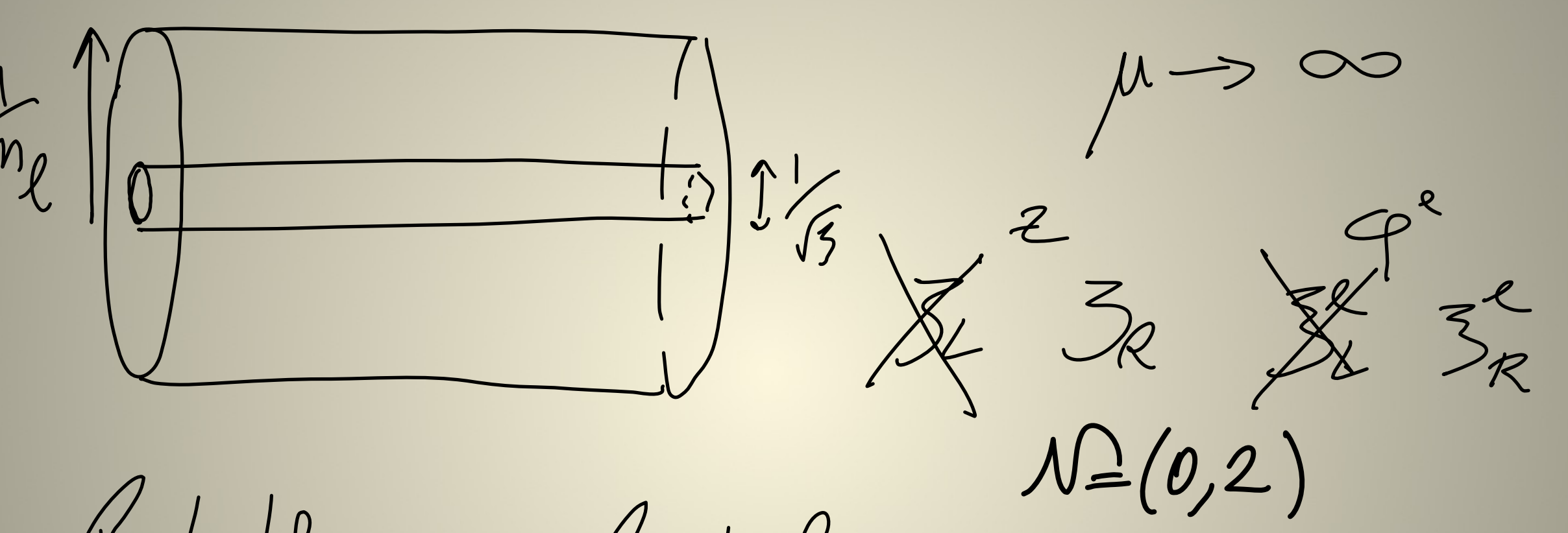

But there are $\underline{\underline{2}}$ problems $\checkmark$ ANomaLY? $\checkmark$ a DEPENDENCE? 
(D): Peculiarities

Everything would be solved if $\Lambda_{C P} \longrightarrow 0$ as $\mu \longrightarrow \infty$ !
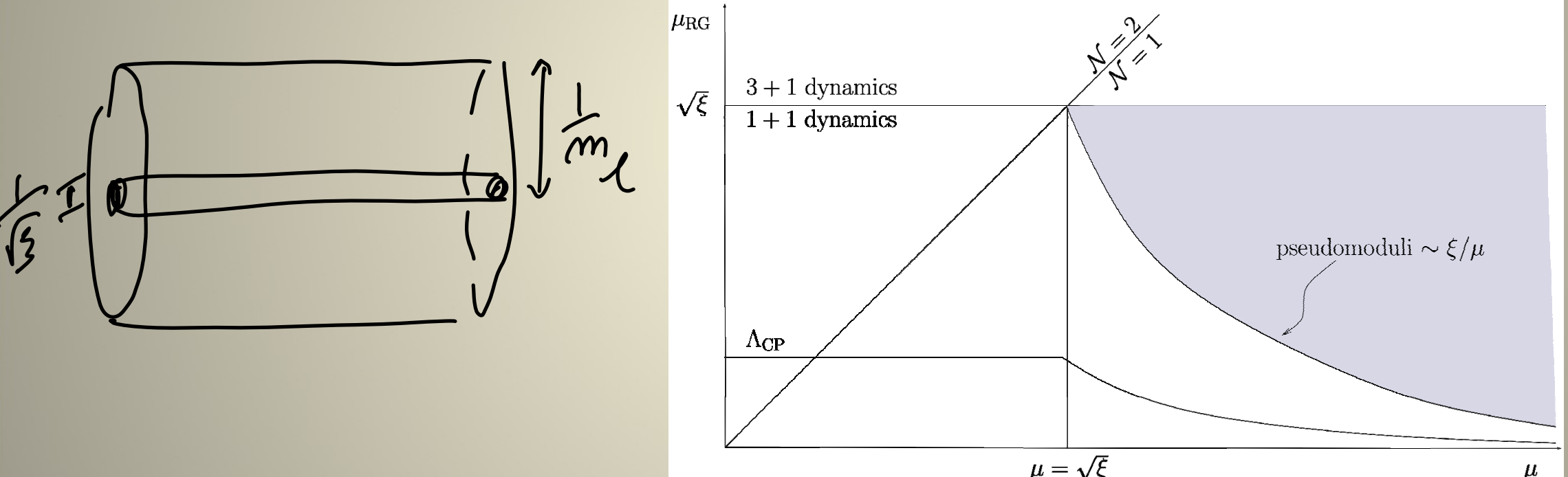

There is entanglement between $3+1 \& 1+1$

$$
S_{t . t}=S_{3+1}+S_{1+1}+S_{\text {int }}
$$


(D): Entanglement: 3+1 with1+1

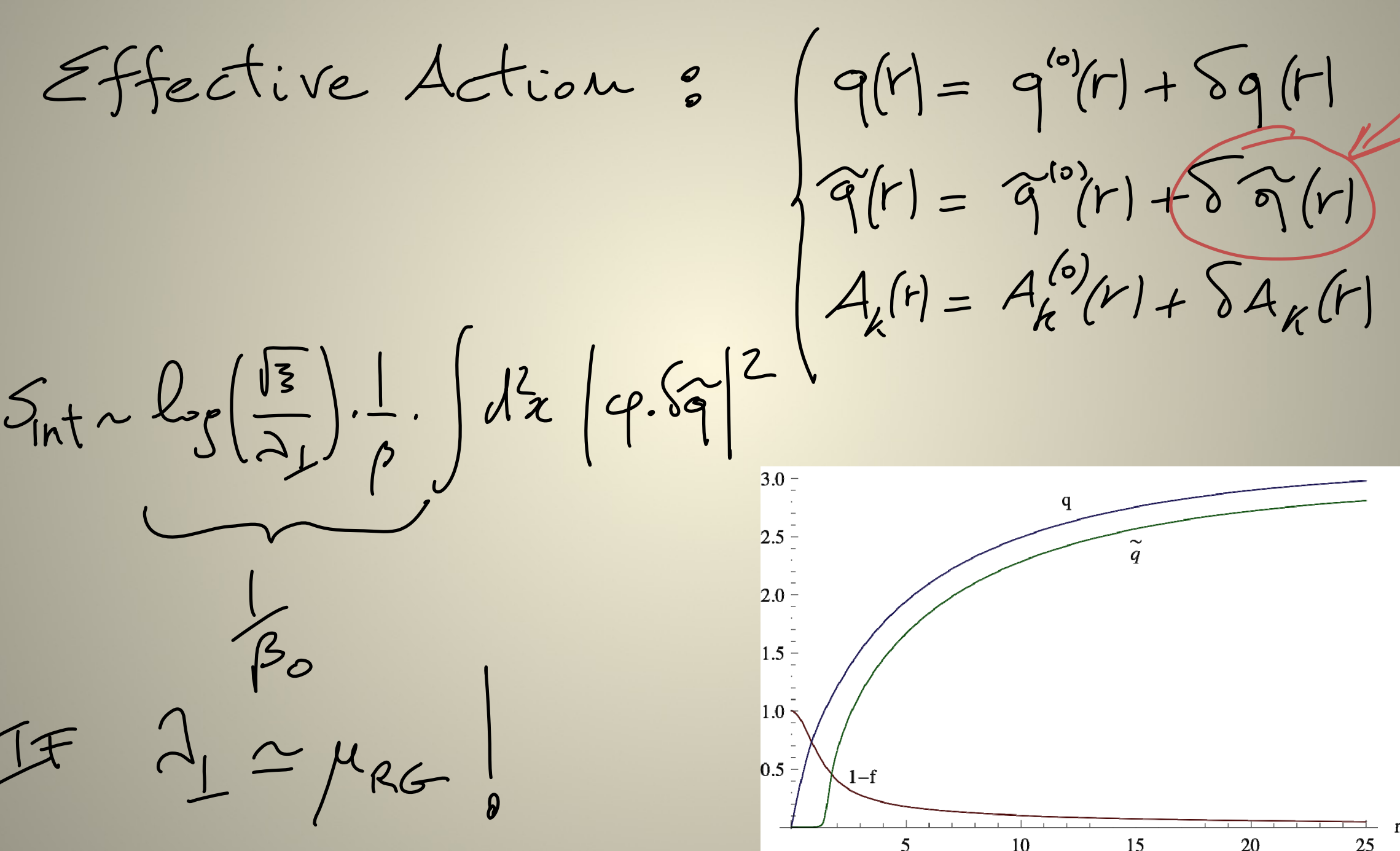


(D): New CFT mechanism

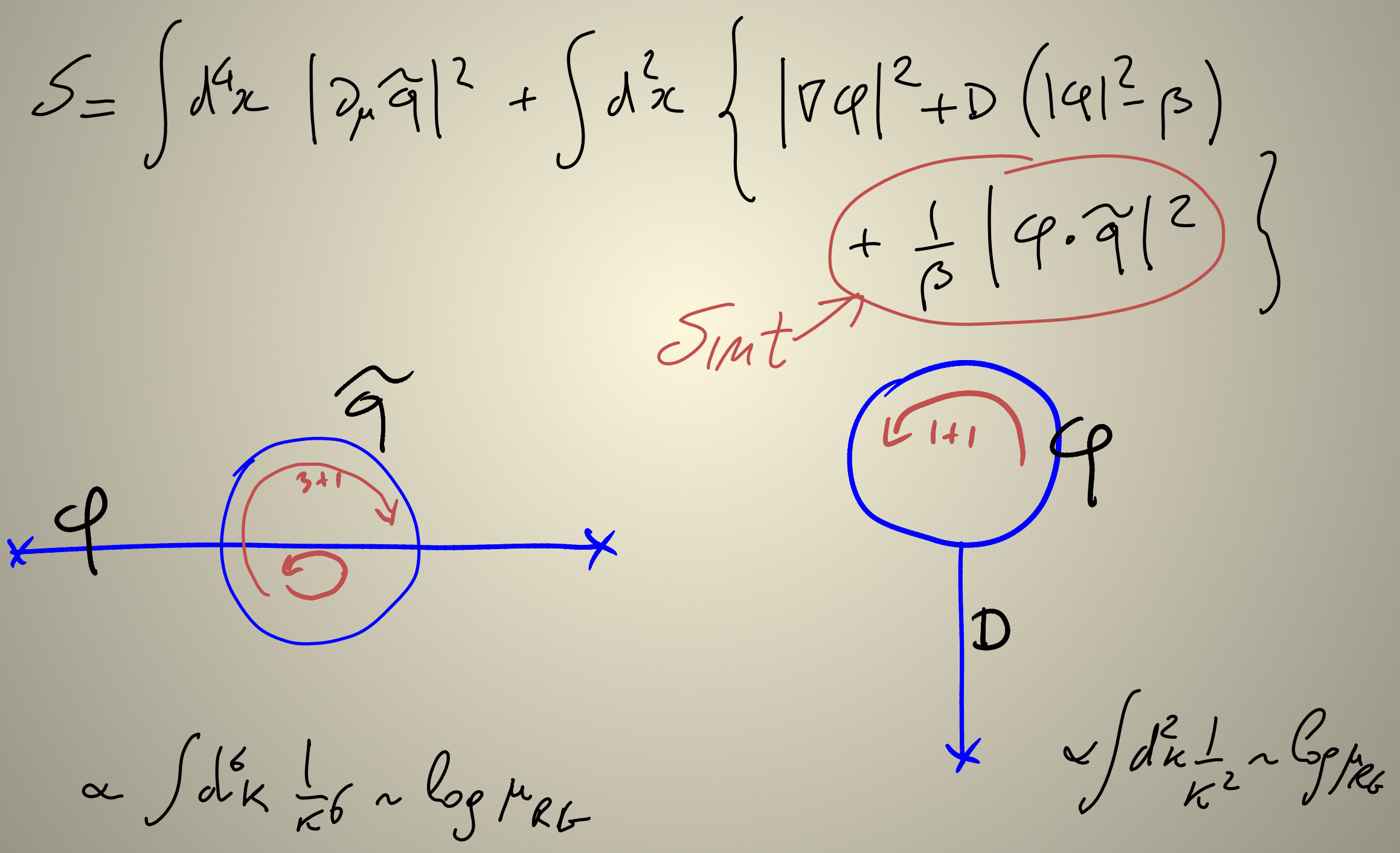


Work in progress ...

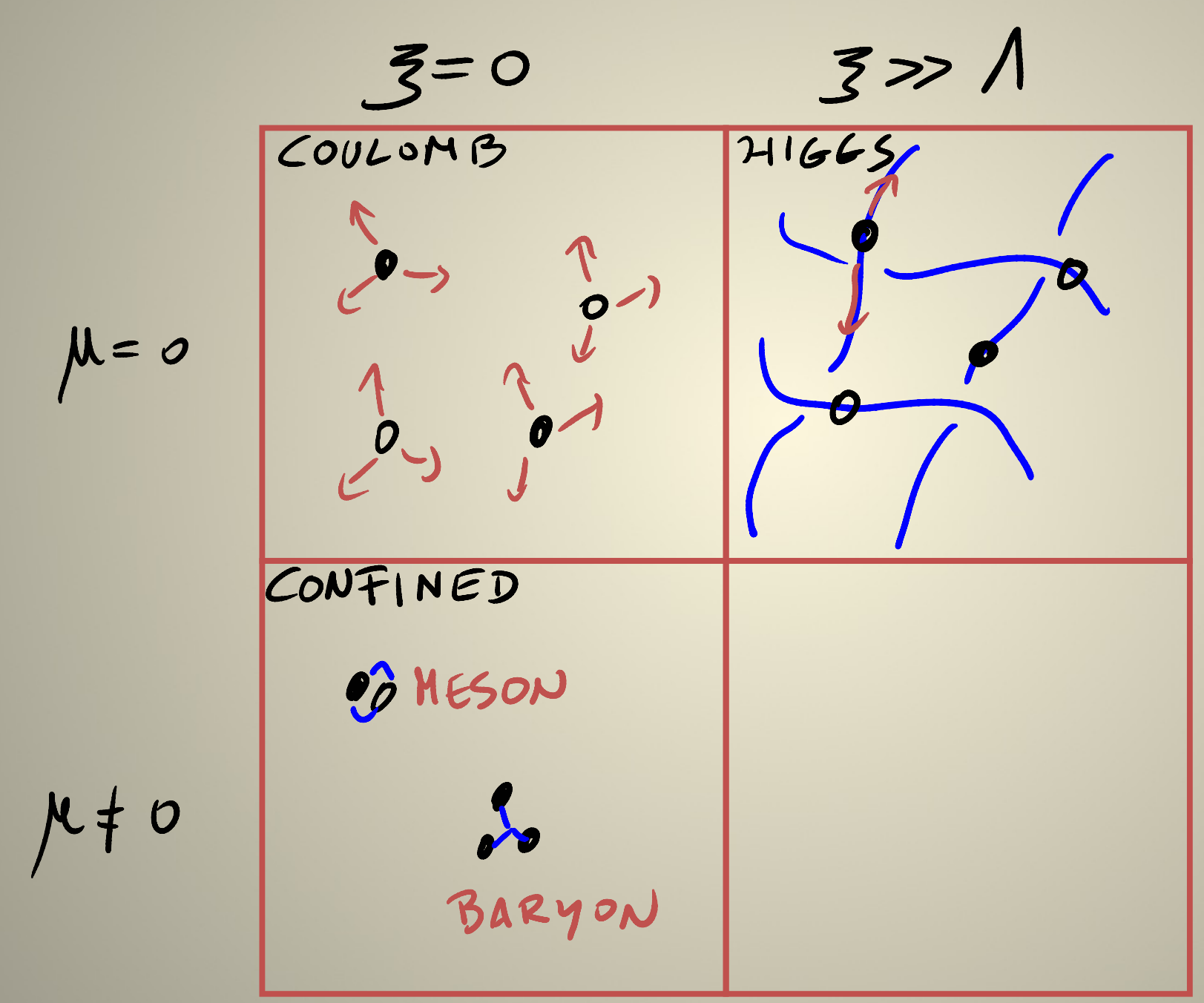


Work in progress ...

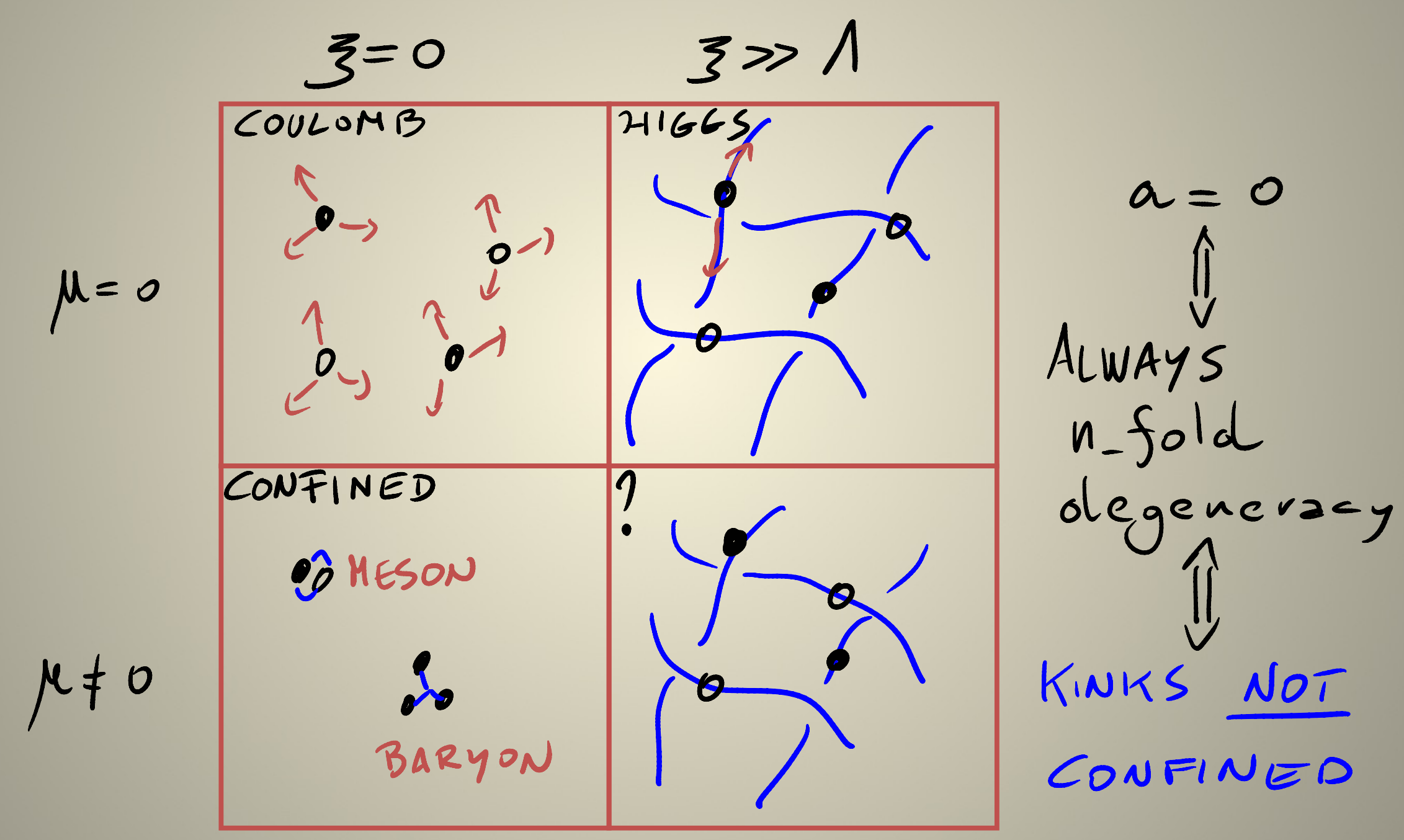


The Idea

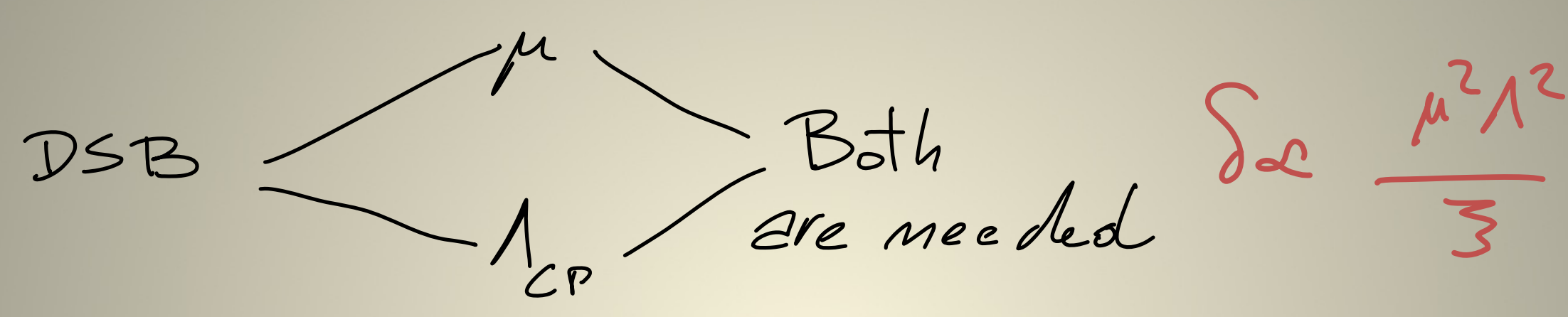


The Idea

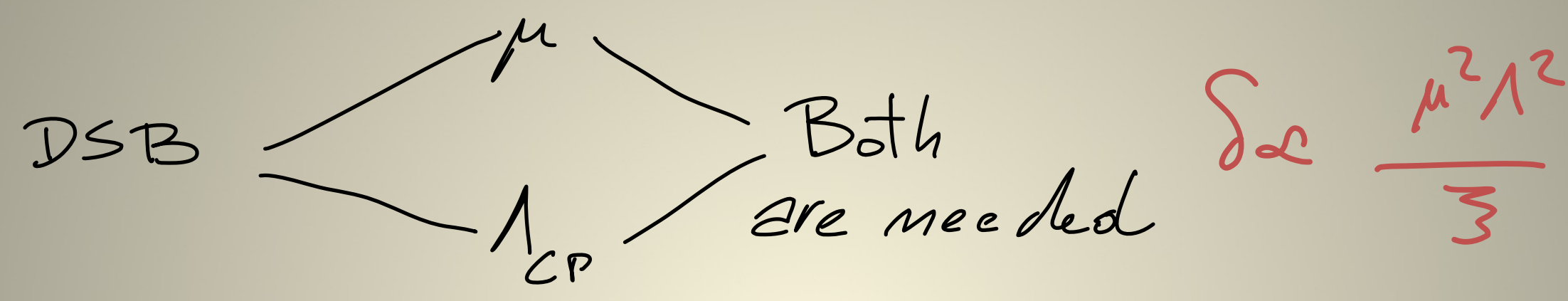

abELIAN Vortex

Global $U(1)$

$q=(\curvearrowleft) \quad A=(\Xi)$

No Internal M. S.

- ${ }_{0}^{0} \sum_{0}^{0}$

$\Rightarrow$ NO DOB! 


\section{Potential}

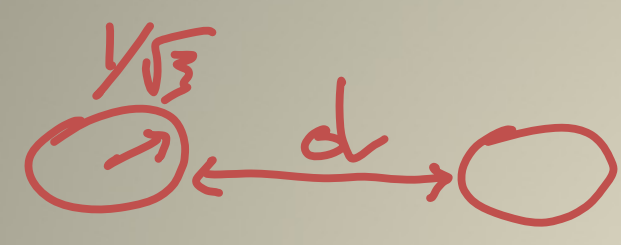

(2)

(III)

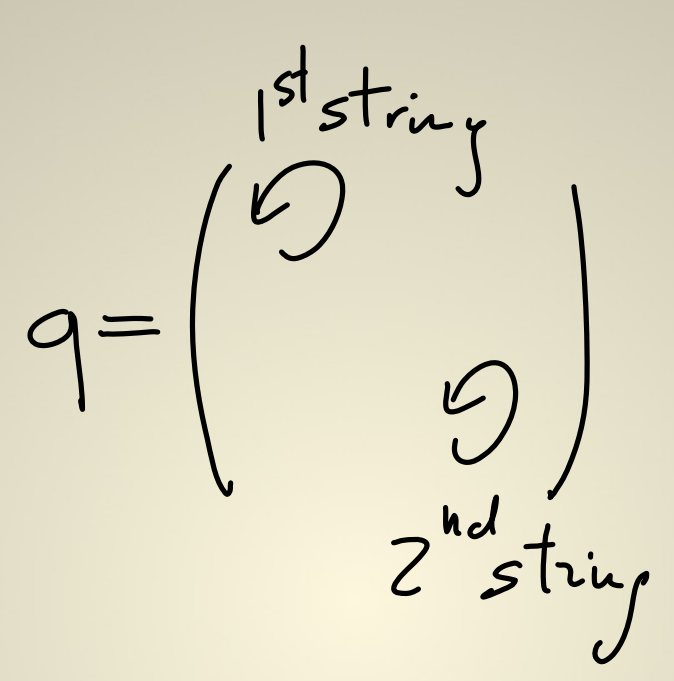

$C(1)--7) \beta$<smiles>O</smiles>

$G(2,2) \cdot 0$ 
Potential

$y / \sqrt{3}$

d

(2)

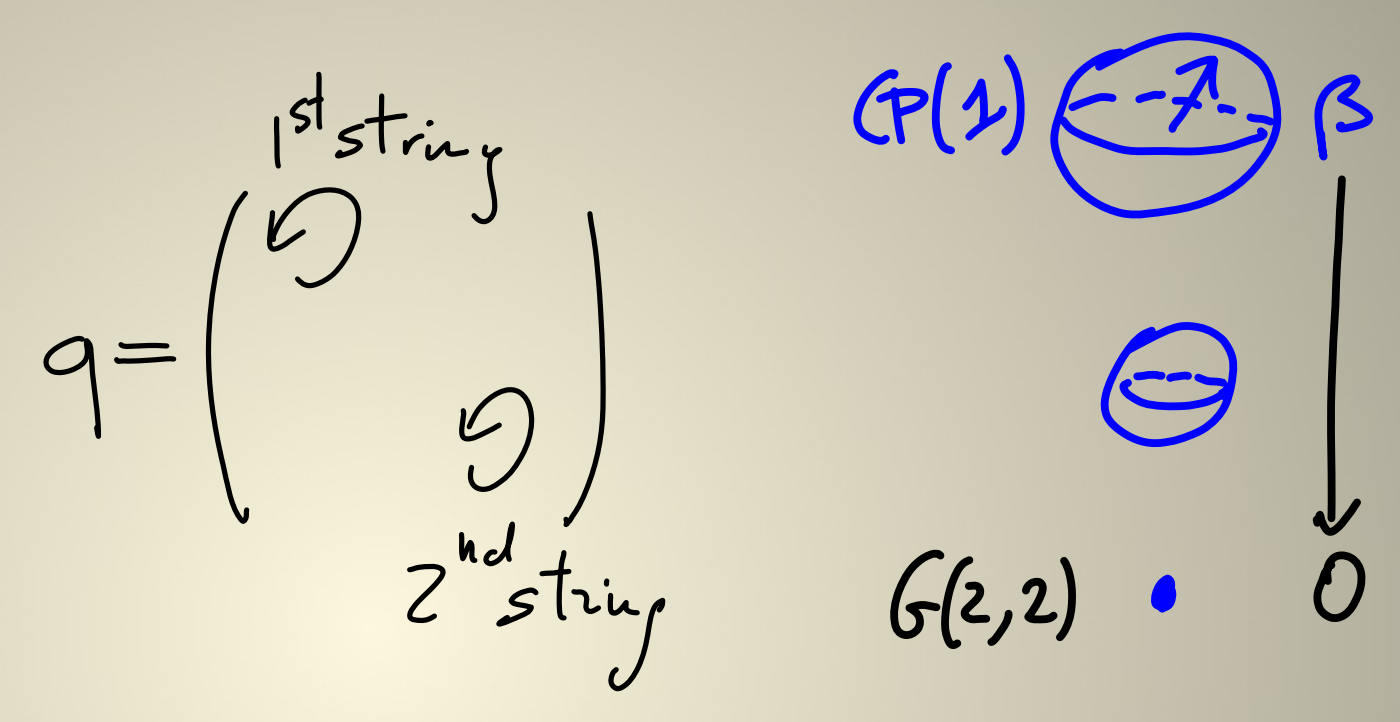

$$
\begin{aligned}
& T_{1}=|\mathbb{Z}|+\delta \\
& T_{2}=2|\mathbb{Z}|
\end{aligned}
$$

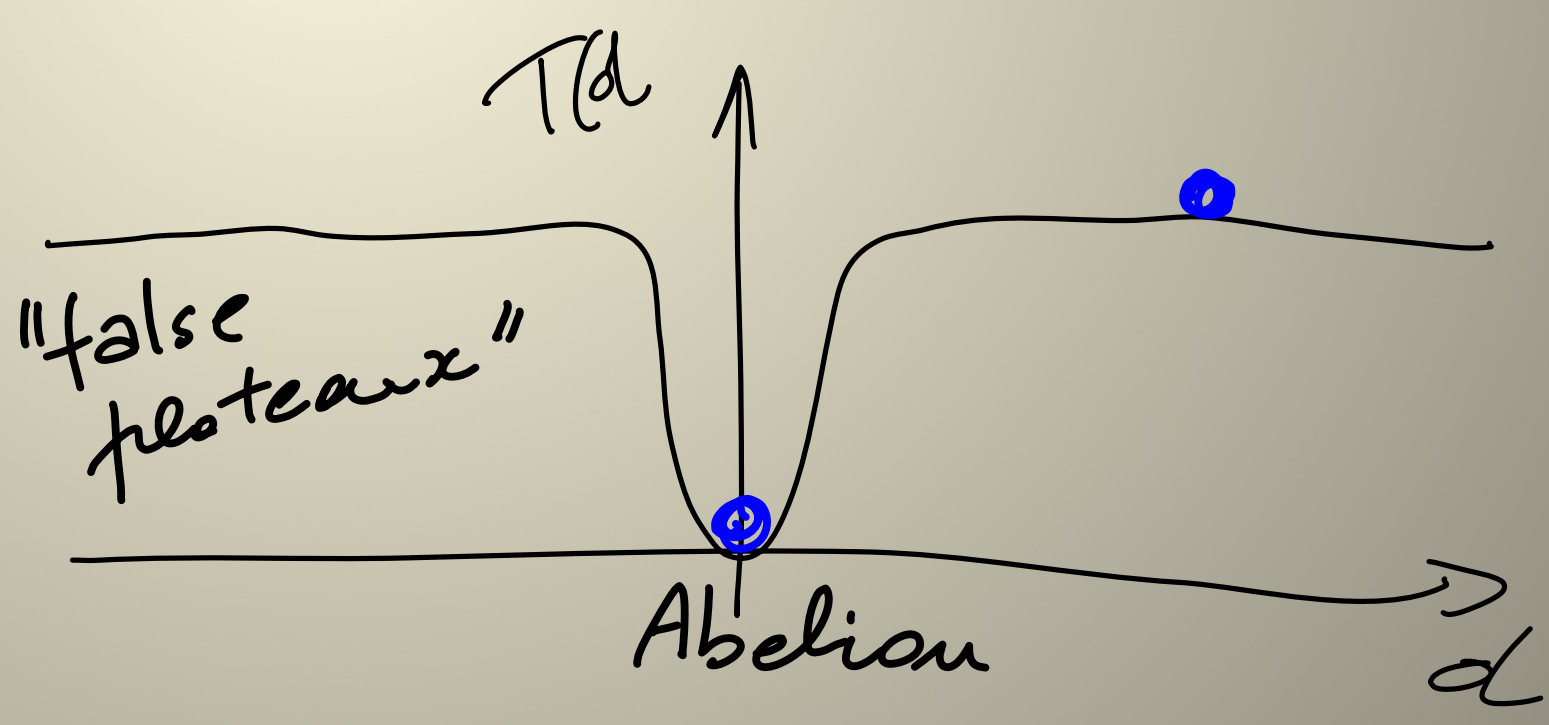


Tunneling<smiles>CCCCCC(CCCC)C(C)C</smiles>

Barrier due to leinetile energy 
Tunneling<smiles>CCCCC(C)C1CCCCC1CCC</smiles>

Barrier due to leinetile energy Bounce Solution

$$
S_{B}=\pi T_{1} \frac{d^{2}}{2 \log \left(R \delta / d^{2}\right)}+\ldots
$$

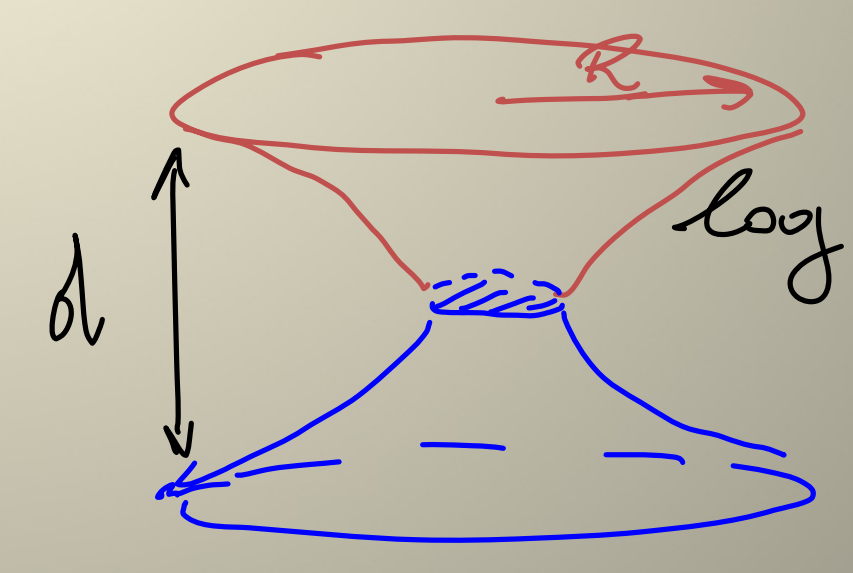


Index

$$
\begin{array}{lcccccccc} 
& Q^{\prime} & Q^{2} & \Phi & Q & Q & \mu & 3 & \Lambda^{2 u_{c}-n_{f}} \\
U(1)_{R} & 1 & 1 & 2 & 0 & 0 & -2 & 0 & 2\left(2 u_{c}-u_{f}\right) \\
U(1)_{J} & 1 & -1 & 0 & 1 & 1 & 2 & 0 & 0 \\
U(1) J^{\prime} & 2 & 0 & 2 & 1 & 1 & \lambda & 0 & 2\left(2 u_{c}-n_{f}\right)
\end{array}
$$

$x \times 0$ STATES

$|v\rangle Q^{\prime}|v\rangle Q^{2}|v\rangle Q^{\prime} Q^{2}|v\rangle$ short in $N=2$

Bot long in $N=1$

$$
v(1)_{j} \mid \rightarrow \mathbb{Z}_{2 n} \rightarrow \mathbb{Z}_{2} \Leftrightarrow D S B
$$




\section{The final experiment ...}

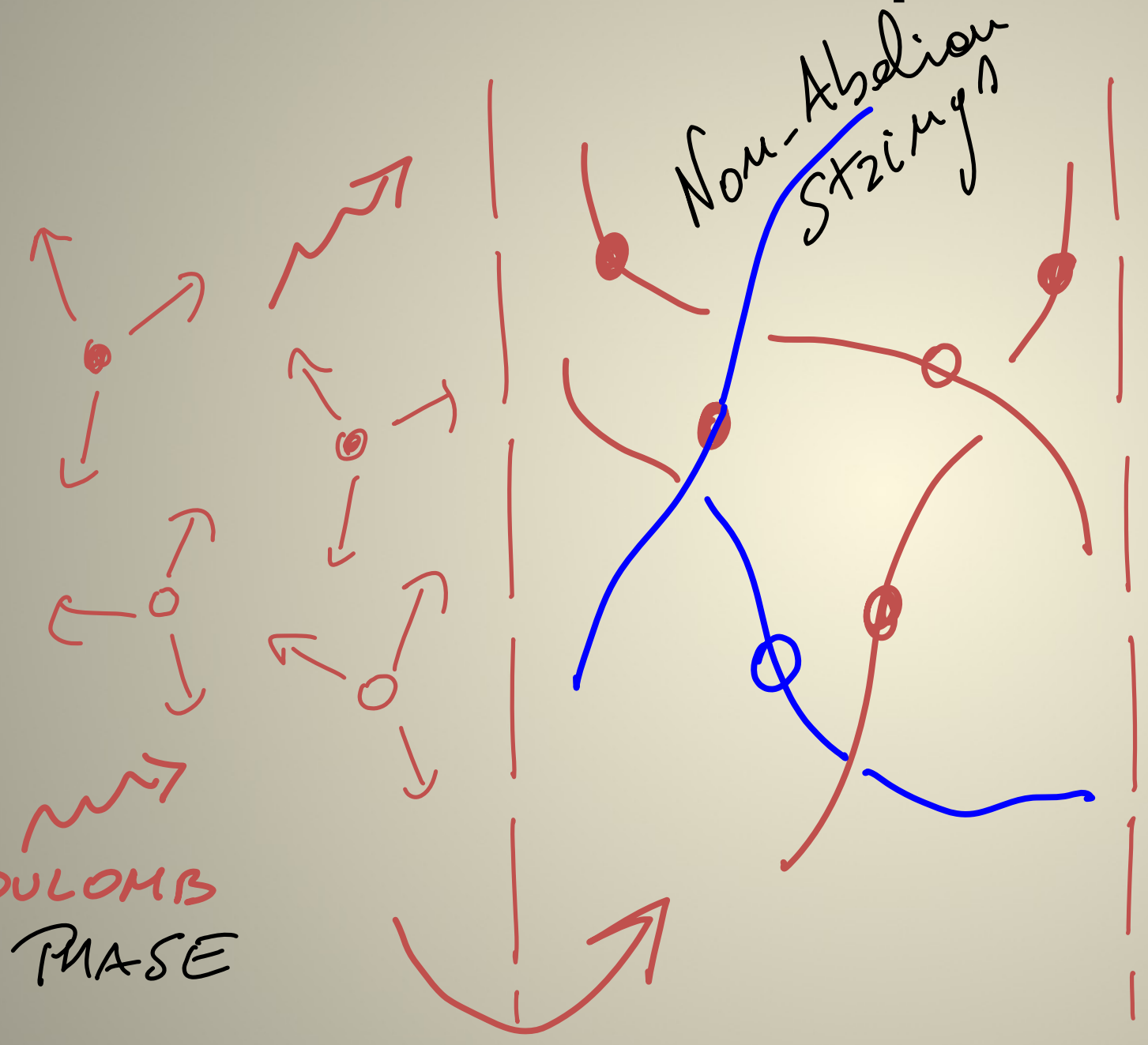

SWITCH OV

$\mu \& \xi$ 
Conclusion

- Studied Heterstic Vortex

$$
w=1, F I, \quad w^{\prime}(m)=0
$$

Explozed $(\mu, \xi)$ porometer spece DSB, nffold deg. " New CFT Q.H.V.

- Abelian bound stote $\begin{aligned} & \text { nemeins BPS } \Rightarrow \text { Phase } \\ & \text { of } S Q C D+F I\end{aligned}$ 